\title{
UPPER AND LOWER BOUNDS FOR LITTLEWOOD-PALEY SQUARE FUNCTIONS IN THE DUNKL SETTING
}

\author{
The present version of the paper extends results of the previous submission and concerns \\ Littlewood-Paley square functions in the Dunkl setting associated with kernels satisfying mild \\ regularity in smoothness and decay.
}

\section{JACEK DZIUBAŃSKI AND AGNIESZKA HEJNA}

Abstract. The aim of this paper is to prove upper and lower $L^{p}$ estimates, $1<p<\infty$, for Littlewood-Paley square functions in the rational Dunkl setting.

\section{IntRoduCtion AND STATEMENTS OF RESUlts}

On $\mathbb{R}^{N}$ equipped with normalized root system $R$ and a multiplicity function $k \geq 0$, let $\nabla f(\mathbf{x})=\left(\partial_{1} f(\mathbf{x}), \partial_{2} f(\mathbf{x}), \ldots, \partial_{N} f(\mathbf{x})\right), \nabla_{k} f(\mathbf{x})=\left(T_{1} f(\mathbf{x}), T_{2} f(\mathbf{x}), \ldots, T_{N} f(\mathbf{x})\right)$, and $\Delta_{k} f(\mathbf{x})=\sum_{j=1}^{\infty} T_{j}^{2} f(\mathbf{x})$ denote the classical gradient, the Dunkl gradient, and the Dunkl Laplacian respectively, where $T_{j}$ are the Dunkl operators (see Section 2). For two reasonable functions $f, g$ on $\mathbb{R}^{N}$, let $f * g$ stands for the Dunkl convolution. Let

$$
f_{t}(\mathbf{x})=t^{-\mathbf{N}} f(\mathbf{x} / t)
$$

where $\mathbf{N}$ is the homogeneous dimension of the system $\left(\mathbb{R}^{N}, R, k\right)$ (see Section 2).

Assume that $\phi$ and $\psi$ are functions defined on $\mathbb{R}^{N}$ which satisfy certain smoothness and decay conditions (see Theorem 1.1 and Corollary 1.3). Assume additionally that $\int_{\mathbb{R}^{N}} \psi d w=0$, where $d w$ is the associated measure (2.2). We define the following square functions:

$$
\begin{aligned}
S_{\nabla_{k}, \phi} f(\mathbf{x}) & =\left(\int_{0}^{\infty}\left|t \nabla_{k}\left(\phi_{t} * f\right)(\mathbf{x})\right|^{2} \frac{d t}{t}\right)^{1 / 2}, \\
S_{\nabla, \phi} f(\mathbf{x}) & =\left(\int_{0}^{\infty}\left|t \nabla\left(\phi_{t} * f\right)(\mathbf{x})\right|^{2} \frac{d t}{t}\right)^{1 / 2}, \\
S_{\psi} f(\mathbf{x}) & =\left(\int_{0}^{\infty}\left|\psi_{t} * f(\mathbf{x})\right|^{2} \frac{d t}{t}\right)^{1 / 2}, \\
S_{\nabla_{t}, \phi} f(\mathbf{x}) & =\left(\int_{0}^{\infty}\left|t \frac{d}{d t}\left(\phi_{t} * f\right)(\mathbf{x})\right|^{2} \frac{d t}{t}\right)^{1 / 2} .
\end{aligned}
$$

For $f, g \in C^{2}\left(\mathbb{R}^{N}\right)$ we consider the carré du champ operator

$$
\Gamma(f, g)=\frac{1}{2}\left(\Delta_{k}(f \bar{g})-f \Delta_{k} \bar{g}-\bar{g} \Delta_{k} f\right)
$$

and the associated square function

$$
\mathfrak{g}_{\Gamma, \phi}(f)(\mathbf{x})=\left(\int_{0}^{\infty} t^{2} \Gamma\left(\phi_{t} * f, \phi_{t} * f\right)(\mathbf{x}) \frac{d t}{t}\right)^{1 / 2} .
$$

2010 Mathematics Subject Classification. primary: 42B25, 42B20; secondary 42B15, 47G10, 47G40.

Key words and phrases. Dunkl operators, Littlewood-Paley square functions, Calderón-Zygmund operators.

Research supported by the National Science Centre, Poland (Narodowe Centrum Nauki), Grant 2017/25/B/ST1/00599. 
Let us note that $\mathfrak{g}_{\Gamma, \phi}$ is well-defined, since $\Gamma(f, f)(\mathbf{x}) \geq 0$ (see (6.2)).

We are now in a position to state our results.

Theorem 1.1. Let $s$ be a positive integer such that $2 s>\mathbf{N}+1$. Assume that $\phi$, $\psi$ are $C^{2 s}\left(\mathbb{R}^{N}\right)$ functions (not necessary radial) such that

$$
\left|\partial^{\beta} \phi(\mathbf{x})\right|+\left|\partial^{\beta} \psi(\mathbf{x})\right| \leq C(1+\|\mathbf{x}\|)^{-M-\mathbf{N}} \quad \text { for } \beta \in \mathbb{N}_{0}^{N},|\beta| \leq 2 s,
$$

for certain $M>\lfloor\mathbf{N}\rfloor+1$. Assume also that $\int_{\mathbb{R}^{N}} \psi d w=0$. Then, for every $1<p<\infty$, there is a constant $C_{p}>0$ such that for all $f \in L^{p}(d w)$ we have

$$
\left\|S_{\nabla_{k}, \phi} f\right\|_{L^{p}(d w)}+\left\|S_{\nabla, \phi} f\right\|_{L^{p}(d w)}+\left\|S_{\psi} f\right\|_{L^{p}(d w)}+\left\|\mathfrak{g}_{\Gamma, \phi}(f)\right\|_{L^{p}(d w)} \leq C_{p}\|f\|_{L^{p}(d w)} .
$$

In order to state lower bounds for the square functions we need additional assumptions on the functions $\phi$ and $\psi$. Let $\mathcal{F}$ denote the Dunkl transform (see (2.11)). We say that the Dunkl transform $\mathcal{F} \phi$ is not identically zero along any direction if

$$
\sup _{t>0}|\mathcal{F} \phi(t \xi)|>0 \quad \text { for every vector } \xi \in \mathbb{R}^{N}, \xi \neq 0 .
$$

This happens if e.g. $\int_{\mathbb{R}^{N}} \phi d w \neq 0$.

Theorem 1.2. Assume that $\phi, \psi$ satisfy the assumptions of Theorem 1.1 and the functions $\mathcal{F} \phi$ and $\mathcal{F} \psi$ are not identically zero along any direction. Then for every $1<p<\infty$ there is a constant $C_{p}>0$ such that for all $f \in L^{p}(d w)$ we have

$$
\begin{aligned}
\|f\|_{L^{p}(d w)} & \leq C_{p}\left\|S_{\nabla_{k}, \phi} f\right\|_{L^{p}(d w)}, \\
\|f\|_{L^{p}(d w)} & \leq C_{p}\left\|S_{\psi} f\right\|_{L^{p}(d w)}, \\
\|f\|_{L^{p}(d w)} & \leq C_{p}\left\|\mathfrak{g}_{\Gamma \phi} f\right\|_{L^{p}(d w)} .
\end{aligned}
$$

Corollary 1.3. Let $s$ be a positive integer such that $2 s>\mathbf{N}+1$. Assume that $\phi \in$ $C^{2 s+1}\left(\mathbb{R}^{N}\right)$ satisfies

$$
\left|\partial^{\beta} \phi(\mathbf{x})\right| \leq C(1+\|\mathbf{x}\|)^{-\mathbf{N}-M-1} \quad \text { for }|\beta| \leq 2 s+1,
$$

for certain $M>\lfloor\mathbf{N}\rfloor+1$. Then, for every $1<p<\infty$, there is a constant $C_{p}>0$ such that for all $f \in L^{p}(d w)$ we have

$$
\left\|S_{\nabla_{t}, \phi} f\right\|_{L^{p}(d w)} \leq C_{p}\|f\|_{L^{p}(d w)} .
$$

If additionally the function $\mathcal{F} \phi$ is not identically zero along any direction, then for every $1<p<\infty$ there is a constant $\widetilde{C}_{p}>0$ such that for all $f \in L^{p}(d w)$ we have

$$
\|f\|_{L^{p}(d w)} \leq \widetilde{C}_{p}\left\|S_{\nabla_{t}, \phi} f\right\|_{L^{p}(d w)} .
$$

In dimension 1 and $\phi(x)=c_{k}^{\prime}\left(1+x^{2}\right)^{-(\mathbf{N}+1) / 2}$, which corresponds to the Poisson semigroup $\exp \left(-t \sqrt{-\Delta_{k}}\right), L^{p}$-bounds of the Littlewood-Paley square functions $S_{\nabla_{k}, \phi}$ and $S_{\nabla_{t}, \phi}$ were studied in [20], [13] and continued in higher dimensions for $1<p \leq 2$ in [21], including the case of $\mathfrak{g}_{\phi}$ in [26]. In the case when $\phi(\mathbf{x})=\exp \left(-\|\mathbf{x}\|^{2}\right)$ and $1<p \leq 2$, the upper and lower bounds for the square function $\mathfrak{g}_{\Gamma, \phi}$ where proved in [14], while the case of $2<p<\infty$ was only considered there for the particular root system, namely when the Coxeter group is isomorphic to $\mathbb{Z}_{2}^{N}$. We want to emphasize that our methods, which allow us to obtain the bounds for the full range of $p$ 's and not necessary radial functions $\phi$ and $\psi$, are different than those of [14]. To prove Theorems 1.1 and 1.2, we adapt techniques of the Calderón-Zygmund analysis to the Dunkl setting (see Section 3). Then, thanks to that, our proofs are reduced to obtaining $L^{2}(d w)$ bounds and verifying that the associated kernels to the square functions satisfy relevant estimates. 


\section{Preliminaries And notation}

The Dunkl theory is a generalization of the Euclidean Fourier analysis. It started with the seminal article [6] and developed extensively afterwards (see e.g. [4], [5], [7], [8], [15], [16], [17], [24]). In this section we present basic facts concerning the theory of the Dunkl operators. For details we refer the reader to [6], [18], and [19].

We consider the Euclidean space $\mathbb{R}^{N}$ with the scalar product $\langle\mathbf{x}, \mathbf{y}\rangle=\sum_{j=1}^{N} x_{j} y_{j}$, $\mathbf{x}=\left(x_{1}, \ldots, x_{N}\right), \mathbf{y}=\left(y_{1}, \ldots, y_{N}\right)$, and the norm $\|\mathbf{x}\|^{2}=\langle\mathbf{x}, \mathbf{x}\rangle$. For a nonzero vector $\alpha \in \mathbb{R}^{N}$, the reflection $\sigma_{\alpha}$ with respect to the hyperplane $\alpha^{\perp}$ orthogonal to $\alpha$ is given by

$$
\sigma_{\alpha}(\mathbf{x})=\mathbf{x}-2 \frac{\langle\mathbf{x}, \alpha\rangle}{\|\alpha\|^{2}} \alpha .
$$

In this paper we fix a normalized root system in $\mathbb{R}^{N}$, that is, a finite set $R \subset \mathbb{R}^{N} \backslash\{0\}$ such that $\sigma_{\alpha}(R)=R$ and $\|\alpha\|=\sqrt{2}$ for every $\alpha \in R$. The finite group $G$ generated by the reflections $\sigma_{\alpha} \in R$ is called the Weyl group (reflection group) of the root system. A multiplicity function is a $G$-invariant function $k: R \rightarrow \mathbb{C}$ which will be fixed and $\geq 0$ throughout this paper. Let

$$
d w(\mathbf{x})=\prod_{\alpha \in R}|\langle\mathbf{x}, \alpha\rangle|^{k(\alpha)} d \mathbf{x}
$$

be the associated measure in $\mathbb{R}^{N}$, where, here and subsequently, $d \mathbf{x}$ stands for the Lebesgue measure in $\mathbb{R}^{N}$. We denote by $\mathbf{N}=N+\sum_{\alpha \in R} k(\alpha)$ the homogeneous dimension of the system. Clearly,

$$
w(B(t \mathbf{x}, t r))=t^{\mathbf{N}} w(B(\mathbf{x}, r)) \text { for all } \mathbf{x} \in \mathbb{R}^{N}, t, r>0
$$

and

$$
\int_{\mathbb{R}^{N}} f(\mathbf{x}) d w(\mathbf{x})=\int_{\mathbb{R}^{N}} t^{-\mathbf{N}} f(\mathbf{x} / t) d w(\mathbf{x}) \text { for } f \in L^{1}(d w) \text { and } t>0 .
$$

Observe that $\left({ }^{1}\right)$

$$
w(B(\mathbf{x}, r)) \sim r^{N} \prod_{\alpha \in R}(|\langle\mathbf{x}, \alpha\rangle|+r)^{k(\alpha)},
$$

so $d w(\mathbf{x})$ is doubling, that is, there is a constant $C>0$ such that

$$
w(B(\mathbf{x}, 2 r)) \leq C w(B(\mathbf{x}, r)) \quad \text { for all } \mathbf{x} \in \mathbb{R}^{N}, r>0 .
$$

Moreover, there exists a constant $C \geq 1$ such that, for every $\mathbf{x} \in \mathbb{R}^{N}$ and for every $r_{2} \geq r_{1}>0$,

$$
C^{-1}\left(\frac{r_{2}}{r_{1}}\right)^{N} \leq \frac{w\left(B\left(\mathbf{x}, r_{2}\right)\right)}{w\left(B\left(\mathbf{x}, r_{1}\right)\right)} \leq C\left(\frac{r_{2}}{r_{1}}\right)^{\mathbf{N}} .
$$

For $\xi \in \mathbb{R}^{N}$, the Dunkl operators $T_{\xi}$ are the following $k$-deformations of the directional derivatives $\partial_{\xi}$ by a difference operator:

$$
T_{\xi} f(\mathbf{x})=\partial_{\xi} f(\mathbf{x})+\sum_{\alpha \in R} \frac{k(\alpha)}{2}\langle\alpha, \xi\rangle \frac{f(\mathbf{x})-f\left(\sigma_{\alpha}(\mathbf{x})\right)}{\langle\alpha, \mathbf{x}\rangle} .
$$

\footnotetext{
${ }^{1}$ The symbol $\sim$ between two positive expressions means that their ratio remains between two positive constants.
} 
The Dunkl operators $T_{\xi}$, which were introduced in [6], commute and are skew-symmetric with respect to the $G$-invariant measure $d w$. For two reasonable functions $f, g$ we have the following integration by parts formula

$$
\int_{\mathbb{R}^{N}} T_{\xi} f(\mathbf{x}) g(\mathbf{x}) d w(\mathbf{x})=-\int_{\mathbb{R}^{N}} f(\mathbf{x}) T_{\xi} g(\mathbf{x}) d w(\mathbf{x}) .
$$

For fixed $\mathbf{y} \in \mathbb{R}^{N}$ the Dunkl kernel $E(\mathbf{x}, \mathbf{y})$ is the unique analytic solution to the system

$$
T_{\xi} f=\langle\xi, \mathbf{y}\rangle f, \quad f(0)=1 .
$$

The function $E(\mathbf{x}, \mathbf{y})$, which generalizes the exponential function $e^{\langle\mathbf{x}, \mathbf{y}\rangle}$, has the unique extension to a holomorphic function on $\mathbb{C}^{N} \times \mathbb{C}^{N}$. Moreover, it satisfies

$$
E(\mathbf{x}, \mathbf{y})=E(\mathbf{y}, \mathbf{x}) \text { and } E(\lambda \mathbf{x}, \mathbf{y})=E(\mathbf{x}, \lambda \mathbf{y})
$$

for all $\mathbf{x}, \mathbf{y} \in \mathbb{C}^{N}$ and $\lambda \in \mathbb{C}$. The following theorem was proved in [16].

Theorem 2.1 ([16, Corollary 5.4]). For all $\mathbf{x}, \mathbf{z} \in \mathbb{R}^{N}$ and $\nu \in \mathbb{N}_{0}^{N}$ we have

$$
\left|\partial_{\mathbf{z}}^{\nu} E(\mathbf{x}, i \mathbf{z})\right| \leq\|\mathbf{x}\|^{|\nu|} \text {. }
$$

Let $\left\{e_{j}\right\}_{1 \leq j \leq N}$ denote the canonical orthonormal basis in $\mathbb{R}^{N}$ and let $T_{j}=T_{e_{j}}$. For multi-index $\beta=\left(\beta_{1}, \beta_{2}, \ldots, \beta_{N}\right) \in \mathbb{N}_{0}^{N}$, we set

$$
\begin{gathered}
|\beta|=\beta_{1}+\beta_{2}+\ldots+\beta_{N}, \\
\partial_{j}^{0}=I, \partial^{\beta}=\partial_{1}^{\beta_{1}} \circ \partial_{2}^{\beta_{2}} \circ \ldots \circ \partial_{N}^{\beta_{N}}, \\
T_{j}^{0}=I, T^{\beta}=T_{1}^{\beta_{1}} \circ T_{2}^{\beta_{2}} \circ \ldots \circ T_{N}^{\beta_{N}} .
\end{gathered}
$$

The Dunkl transform

$$
\mathcal{F} f(\xi)=c_{k}^{-1} \int_{\mathbb{R}^{N}} E(-i \xi, \mathbf{x}) f(\mathbf{x}) d w(\mathbf{x})
$$

where

$$
c_{k}=\int_{\mathbb{R}^{N}} e^{-\frac{\|\mathbf{x}\|^{2}}{2}} d w(\mathbf{x})>0,
$$

originally defined for $f \in L^{1}(d w)$, is an isometry on $L^{2}(d w)$, i.e.,

$$
\|f\|_{L^{2}(d w)}=\|\mathcal{F} f\|_{L^{2}(d w)} \text { for all } f \in L^{2}(d w),
$$

and preserves the Schwartz class of functions $\mathcal{S}\left(\mathbb{R}^{N}\right)$ (see [3]). Its inverse $\mathcal{F}^{-1}$ has the form

$$
\mathcal{F}^{-1} g(\mathbf{x})=c_{k}^{-1} \int_{\mathbb{R}^{N}} E(i \xi, \mathbf{x}) g(\xi) d w(\xi)
$$

Moreover,

$$
\mathcal{F}\left(T_{j} f\right)(\xi)=i \xi_{j} \mathcal{F} f(\xi) .
$$

The Dunkl translation $\tau_{\mathbf{x}} f$ of a function $f \in \mathcal{S}\left(\mathbb{R}^{N}\right)$ by $\mathbf{x} \in \mathbb{R}^{N}$ is defined by

$$
\tau_{\mathbf{x}} f(\mathbf{y})=c_{k}^{-1} \int_{\mathbb{R}^{N}} E(i \xi, \mathbf{x}) E(i \xi, \mathbf{y}) \mathcal{F} f(\xi) d w(\xi) .
$$

It is a contraction on $L^{2}(d w)$, however it is an open problem if the Dunkl translations are bounded operators on $L^{p}(d w)$ for $p \neq 2$. 
The Dunkl convolution $f * g$ of two reasonable functions (for instance Schwartz functions) is defined by

$$
(f * g)(\mathbf{x})=c_{k} \mathcal{F}^{-1}[(\mathcal{F} f)(\mathcal{F} g)](\mathbf{x})=\int_{\mathbb{R}^{N}}(\mathcal{F} f)(\xi)(\mathcal{F} g)(\xi) E(\mathbf{x}, i \xi) d w(\xi) \text { for } \mathbf{x} \in \mathbb{R}^{N}
$$

or, equivalently, by

$$
(f * g)(\mathbf{x})=\int_{\mathbb{R}^{N}} f(\mathbf{y}) \tau_{\mathbf{x}} g(-\mathbf{y}) d w(\mathbf{y})=\int_{\mathbb{R}^{N}} f(\mathbf{y}) g(\mathbf{x}, \mathbf{y}) d w(\mathbf{y}) \text { for all } \mathbf{x} \in \mathbb{R}^{N},
$$

where, here and subsequently,

$$
g(\mathbf{x}, \mathbf{y})=\tau_{\mathbf{x}} g(-\mathbf{y})=\tau_{-\mathbf{y}} g(\mathbf{x})=g(-\mathbf{y},-\mathbf{x}) .
$$

Let us point out that it is not known if the Young inequality $\|f * g\|_{L^{p}(d w)} \leq C\|f\|_{L^{1}(d w)}\|g\|_{L^{p}(d w)}$ holds in the Dunkl setting, unless $p=2$ or $G=\mathbb{Z}_{2}^{N}$ or one of the functions is radial.

The Dunkl Laplacian associated with $R$ and $k$ is the differential-difference operator $\Delta_{k}=\sum_{j=1}^{N} T_{j}^{2}$, which acts on $C^{2}\left(\mathbb{R}^{N}\right)$-functions by

$$
\begin{gathered}
\Delta_{k} f(\mathbf{x})=\Delta_{\text {eucl }} f(\mathbf{x})+\sum_{\alpha \in R} k(\alpha) \delta_{\alpha} f(\mathbf{x}), \\
\delta_{\alpha} f(\mathbf{x})=\frac{\partial_{\alpha} f(\mathbf{x})}{\langle\alpha, \mathbf{x}\rangle}-\frac{\|\alpha\|^{2}}{2} \frac{f(\mathbf{x})-f\left(\sigma_{\alpha} \mathbf{x}\right)}{\langle\alpha, \mathbf{x}\rangle^{2}} .
\end{gathered}
$$

Obviously, $\mathcal{F}\left(\Delta_{k} f\right)(\xi)=-\|\xi\|^{2} \mathcal{F} f(\xi)$. The operator $\Delta_{k}$ is essentially self-adjoint on $L^{2}(d w)$ (see for instance [2, Theorem 3.1]) and generates the semigroup $e^{t \Delta_{k}}$ of linear self-adjoint contractions on $L^{2}(d w)$. The semigroup has the form

$$
e^{t \Delta_{k}} f(\mathbf{x})=\mathcal{F}^{-1}\left(e^{-t\|\xi\|^{2}} \mathcal{F} f(\xi)\right)(\mathbf{x})=\int_{\mathbb{R}^{N}} h_{t}(\mathbf{x}, \mathbf{y}) f(\mathbf{y}) d w(\mathbf{y}),
$$

where the heat kernel

$$
\begin{gathered}
h_{t}(\mathbf{x}, \mathbf{y})=\tau_{\mathbf{x}} h_{t}(-\mathbf{y}) \\
h_{t}(\mathbf{x})=\mathcal{F}^{-1}\left(e^{-t\|\xi\|^{2}}\right)(\mathbf{x})=c_{k}^{-1}(2 t)^{-\mathbf{N} / 2} e^{-\|\mathbf{x}\|^{2} /(4 t)}
\end{gathered}
$$

is a $C^{\infty}$-function of all variables $\mathbf{x}, \mathbf{y} \in \mathbb{R}^{N}, t>0$, and satisfies

$$
\begin{gathered}
0<h_{t}(\mathbf{x}, \mathbf{y})=h_{t}(\mathbf{y}, \mathbf{x}), \\
\int_{\mathbb{R}^{N}} h_{t}(\mathbf{x}, \mathbf{y}) d w(\mathbf{y})=1 .
\end{gathered}
$$

Set

$$
d(\mathbf{x}, \mathbf{y})=\min _{\sigma \in G}\|\mathbf{x}-\sigma(\mathbf{y})\|
$$

$$
V(\mathbf{x}, \mathbf{y}, t)=\max (w(B(\mathbf{x}, t)), w(B(\mathbf{y}, t))), \quad \mathcal{V}(\mathbf{x}, \mathbf{y}, t)=w(B(\mathbf{x}, t))^{1 / 2} w(B(\mathbf{y}, t))^{1 / 2} .
$$

Note that by (2.4) and (2.5) we have

$$
V(\mathbf{x}, \mathbf{y}, d(\mathbf{x}, \mathbf{y})) \sim \mathcal{V}(\mathbf{x}, \mathbf{y}, d(\mathbf{x}, \mathbf{y})) \sim w(B(\mathbf{x}, d(\mathbf{x}, \mathbf{y}))) \sim w(B(\mathbf{y}, d(\mathbf{x}, \mathbf{y}))) .
$$

The following theorem was proved in [1, Theorem 4.1]. 
Theorem 2.2. (a) Time derivatives: for any non-negative integer $m$, there are constants $C, c>0$ such that

$$
\left|\partial_{t}^{m} h_{t}(\mathbf{x}, \mathbf{y})\right| \leq C t^{-m} V(\mathbf{x}, \mathbf{y}, \sqrt{t})^{-1} e^{-c d(\mathbf{x}, \mathbf{y})^{2} / t},
$$

for every $t>0$ and for every $\mathbf{x}, \mathbf{y} \in \mathbb{R}^{N}$.

(b) Hölder bounds: for any nonnegative integer $m$, there are constants $C, c>0$ such that

$$
\left|\partial_{t}^{m} h_{t}(\mathbf{x}, \mathbf{y})-\partial_{t}^{m} h_{t}\left(\mathbf{x}, \mathbf{y}^{\prime}\right)\right| \leq C t^{-m}\left(\frac{\left\|\mathbf{y}-\mathbf{y}^{\prime}\right\|}{\sqrt{t}}\right) V(\mathbf{x}, \mathbf{y}, \sqrt{t})^{-1} e^{-c d(\mathbf{x}, \mathbf{y})^{2} / t}
$$

for every $t>0$ and for every $\mathbf{x}, \mathbf{y}, \mathbf{y}^{\prime} \in \mathbb{R}^{N}$ such that $\left\|\mathbf{y}-\mathbf{y}^{\prime}\right\|<\sqrt{t}$.

(c) Dunkl derivative: for any $\xi \in \mathbb{R}^{N}$ and for any nonnegative integer $m$, there are constants $C, c>0$ such that

$$
\left|T_{\xi, \mathbf{x}} \partial_{t}^{m} h_{t}(\mathbf{x}, \mathbf{y})\right| \leq C t^{-m-1 / 2} V(\mathbf{x}, \mathbf{y}, \sqrt{t})^{-1} e^{-c d(\mathbf{x}, \mathbf{y})^{2} / t},
$$

for all $t>0$ and $\mathbf{x}, \mathbf{y} \in \mathbb{R}^{N}$.

(d) Mixed derivatives: for any nonnegative integer $m$ and for any multi-indices $\alpha, \beta$, there are constants $C, c>0$ such that, for every $t>0$ and for every $\mathbf{x}, \mathbf{y} \in \mathbb{R}^{N}$,

$$
\left|\partial_{t}^{m} \partial_{\mathbf{x}}^{\alpha} \partial_{\mathbf{y}}^{\beta} h_{t}(\mathbf{x}, \mathbf{y})\right| \leq C t^{-m-\frac{|\alpha|}{2}-\frac{|\beta|}{2}} V(\mathbf{x}, \mathbf{y}, \sqrt{t})^{-1} e^{-c d(\mathbf{x}, \mathbf{y})^{2} / t},
$$

for every $t>0$ and for every $\mathbf{x}, \mathbf{y} \in \mathbb{R}^{N}$.

We finish this section by the proposition which will be used in proving the bounds of the square functions.

Proposition 2.3. Let $M \geq 0$ and let $\ell$ be a positive integer. Assume that $\phi \in C^{\ell}\left(\mathbb{R}^{N}\right)$ satisfies

$$
\left|\partial^{\beta} \phi(\mathbf{x})\right| \leq(1+\|\mathbf{x}\|)^{-\mathbf{N}-M} \text { for all }|\beta| \leq \ell
$$

There is a constant $C>0$ such that

$$
\left|T^{\beta} \phi(\mathbf{x})\right| \leq C(1+\|\mathbf{x}\|)^{-\mathbf{N}-M} \text { for all }|\beta| \leq \ell .
$$

Moreover, if additionally $M>0$ and $m$ is a positive integer such that $m<M$, then $\mathcal{F} \phi \in C^{m}\left(\mathbb{R}^{N}\right)$ and

$$
\left|\partial_{\xi}^{\beta^{\prime}} \mathcal{F} \phi(\xi)\right| \leq C_{\beta^{\prime}}(1+\|\xi\|)^{-\ell} \quad \text { for all }\left|\beta^{\prime}\right| \leq m .
$$

Proof. The proof of (2.25) is by induction on $\ell$. Assume that (2.24) implies (2.25) for $\ell_{1}$. We will prove that (2.24) implies (2.25) for $\ell_{1}+1$. Let $\alpha \in R$. By the definition of $T_{j}$, it is enough to show that there is a constant $C>0$ such that the function

$$
\mathbf{x} \longmapsto C^{-1} \frac{\phi(\mathbf{x})-\phi\left(\sigma_{\alpha}(\mathbf{x})\right)}{\langle\mathbf{x}, \alpha\rangle}
$$

satisfies (2.24) with $\ell=\ell_{1}$. Let $\beta \in \mathbb{N}_{0}^{N}$ be such that $|\beta| \leq \ell_{1}$. We consider two cases. Case 1. $|\langle\mathbf{x}, \alpha\rangle| \geq 1 / 10$. Note that there is a constant $C=C_{\ell_{1}}>0$ independent of $\mathbf{x}$ such that for all $\left|\beta^{\prime}\right| \leq \ell_{1}$ we have

$$
\left|\partial_{\mathbf{x}}^{\beta^{\prime}}\left(\langle\mathbf{x}, \alpha\rangle^{-1}\right)\right| \leq C .
$$


Thanks to the Leibniz rule, the estimate for $\partial^{\beta}\left(\frac{\phi(\mathbf{x})-\phi\left(\sigma_{\alpha}(\mathbf{x})\right)}{\langle\mathbf{x}, \alpha\rangle}\right)$ is a consequence of $(2.24)$ and (2.27).

Case 2. $|\langle\mathbf{x}, \alpha\rangle|<1 / 10$. We have

$$
\begin{aligned}
\frac{\phi(\mathbf{x})-\phi\left(\sigma_{\alpha}(\mathbf{x})\right)}{\langle\mathbf{x}, \alpha\rangle} & =\langle\mathbf{x}, \alpha\rangle^{-1} \int_{0}^{1} \frac{d}{d t}\left(\phi\left(\mathbf{x}-2 t \alpha\|\alpha\|^{-2}\langle\mathbf{x}, \alpha\rangle\right)\right) d t \\
& =c_{\alpha} \int_{0}^{1}\left\langle\nabla_{\mathbf{x}} \phi\left(\mathbf{x}-2 t \alpha\|\alpha\|^{-2}\langle\mathbf{x}, \alpha\rangle\right), \alpha\right\rangle d t
\end{aligned}
$$

By the assumption $|\langle\mathbf{x}, \alpha\rangle|<1 / 10$, so for all $t \in[0,1]$ we have

$$
\|\mathbf{x}-2 t \alpha\| \alpha\left\|^{-2}\langle\mathbf{x}, \alpha\rangle\right\| \sim\|\mathbf{x}\|
$$

so, by (2.24) with $\ell=\ell_{1}+1$ we obtain

$$
\begin{aligned}
& \left|\partial_{\mathbf{x}}^{\beta}\left(\frac{\phi(\mathbf{x})-\phi\left(\sigma_{\alpha}(\mathbf{x})\right)}{\langle\mathbf{x}, \alpha\rangle}\right)\right| \leq\left|c_{\alpha} \int_{0}^{1}\left\langle\partial_{\mathbf{x}}^{\beta} \nabla_{\mathbf{x}} \phi\left(\mathbf{x}-2 t \alpha\|\alpha\|^{-2}\langle\mathbf{x}, \alpha\rangle\right), \alpha\right\rangle d t\right| \\
& \leq C \int_{0}^{1}\left(1+\|\mathbf{x}-2 t \alpha\| \alpha\left\|^{-2}\langle\mathbf{x}, \alpha\rangle\right\|\right)^{-\mathbf{N}-M} d t \leq C(1+\|\mathbf{x}\|)^{-\mathbf{N}-M}
\end{aligned}
$$

which completes the proof of (2.25).

We now turn to prove (2.26). By (2.10) and Theorem 2.1 we have $\left|\partial_{\xi}^{\beta} E(-i \mathbf{x}, \xi)\right| \leq$ $C\|\mathbf{x}\|^{|\beta|}$. Thus, using (2.13), we obtain

$$
\begin{aligned}
\left|\partial_{\xi}^{\beta^{\prime}}\left(\xi^{\beta} \mathcal{F} \phi(\xi)\right)\right| & =c_{k}^{-1}\left|\int_{\mathbb{R}^{N}}\left(T^{\beta} \phi\right)(\mathbf{x}) \partial_{\xi}^{\beta^{\prime}} E(-i \mathbf{x}, \xi) d w(\mathbf{x})\right| \\
& \leq C \int_{\mathbb{R}^{N}}(1+\|\mathbf{x}\|)^{-M-\mathbf{N}}(1+\|\mathbf{x}\|)^{m} d w(\mathbf{x}) \leq C_{\beta^{\prime}, \beta}
\end{aligned}
$$

for $\left|\beta^{\prime}\right| \leq m$ and $|\beta| \leq \ell$. From (2.30) we conclude that for $\left|\beta^{\prime}\right| \leq m$ and $|\beta| \leq \ell$ one has

$$
\left|\xi^{\beta} \partial_{\xi}^{\beta^{\prime}} \mathcal{F} \phi(\xi)\right| \leq C_{\beta^{\prime}, \beta}^{\prime}
$$

Now (2.26) can be deduced easily from (2.31).

\section{Vector valued CAlderón-Zygmund anAlysis in the Dunkl Setting}

The proof of main results will be based on the following straightforward adaptation of the vector valued approach to square functions in the Dunkl setting (cf. [9], [22]). Since the conditions on kernels are expressed by means of both: the Euclidean distance $\|\mathbf{x}-\mathbf{y}\|$ and the distance $d(\mathbf{x}, \mathbf{y})$, for the convenience of the reader we present the details.

3.1. Vector valued Calderón-Zygmund operators in the Dunkl setting. Let $\mathcal{H}_{1}$ and $\mathcal{H}_{2}$ be separable Hilbert spaces. We shall consider the vector valued $L^{p}\left(d w, \mathcal{H}_{j}\right)$ spaces with the norms

$$
\|f\|_{L^{p}\left(d w, \mathcal{H}_{j}\right)}^{p}=\int_{\mathbb{R}^{N}}\|f(\mathbf{x})\|_{\mathcal{H}_{j}}^{p} d w(\mathbf{x}) .
$$

Note that $L^{2}\left(d w, \mathcal{H}_{j}\right)=: \mathbf{H}_{j}$ is a Hilbert space with the inner product

$$
\langle f, g\rangle_{\mathbf{H}_{j}}=\int_{\mathbb{R}^{N}}\langle f(\mathbf{x}), g(\mathbf{x})\rangle_{\mathcal{H}_{j}} d w(\mathbf{x}) .
$$


Proposition 3.1. Let $\mathcal{K}$ be a bounded linear operator from $L^{2}\left(d w, \mathcal{H}_{1}\right)$ into $L^{2}\left(d w, \mathcal{H}_{2}\right)$ with an associated operator valued kernel $\mathcal{K}(\mathbf{x}, \mathbf{y}) \in \mathcal{L}\left(\mathcal{H}_{1}, \mathcal{H}_{2}\right)$ for $d(\mathbf{x}, \mathbf{y})>0$. Assume that there are constants $C, \delta^{\prime}>0$ such that for all $\mathbf{x}, \mathbf{y}, \mathbf{y}^{\prime} \in \mathbb{R}^{N}$ such that $2\left\|\mathbf{y}-\mathbf{y}^{\prime}\right\| \leq$ $d(\mathbf{x}, \mathbf{y})$ one has

$$
\begin{aligned}
& \left\|\mathcal{K}(\mathbf{x}, \mathbf{y})-\mathcal{K}\left(\mathbf{x}, \mathbf{y}^{\prime}\right)\right\|_{\mathcal{L}\left(\mathcal{H}_{1}, \mathcal{H}_{2}\right)} \leq \frac{C}{V(\mathbf{x}, \mathbf{y}, d(\mathbf{x}, \mathbf{y}))}\left(\frac{\left\|\mathbf{y}-\mathbf{y}^{\prime}\right\|}{d(\mathbf{x}, \mathbf{y})}\right)^{\delta^{\prime}}, \\
& \left\|\mathcal{K}(\mathbf{y}, \mathbf{x})-\mathcal{K}\left(\mathbf{y}^{\prime}, \mathbf{x}\right)\right\|_{\mathcal{L}\left(\mathcal{H}_{1}, \mathcal{H}_{2}\right)} \leq \frac{C}{V(\mathbf{x}, \mathbf{y}, d(\mathbf{x}, \mathbf{y}))}\left(\frac{\left\|\mathbf{y}-\mathbf{y}^{\prime}\right\|}{d(\mathbf{x}, \mathbf{y})}\right)^{\delta^{\prime}} .
\end{aligned}
$$

Then, for every $1<p<\infty$, the operator $\mathcal{K}$, initially defined on $L^{p}\left(d w, \mathcal{H}_{1}\right) \cap L^{2}\left(d w, \mathcal{H}_{1}\right)$, has a unique extension to a bounded operator from $L^{p}\left(d w, \mathcal{H}_{1}\right)$ to $L^{p}\left(d w, \mathcal{H}_{2}\right)$.

Proof. First we prove the weak type $(1,1)$ estimate. Consider $f \in L^{1}\left(d w, \mathcal{H}_{1}\right)$. Fix $\lambda>0$. We denote by $\mathcal{Q}_{\lambda}$ the collection of all maximal (disjoint) dyadic cubes $Q_{j}$ in $\mathbb{R}^{N}$ satisfying

$$
\lambda<\frac{1}{w\left(Q_{j}\right)} \int_{Q_{j}}\|f(\mathbf{x})\|_{\mathcal{H}_{1}} d w(\mathbf{x}) .
$$

Then, thanks to (2.5), we have

$$
\frac{1}{w\left(Q_{j}\right)} \int_{Q_{j}}\|f(\mathbf{x})\|_{\mathcal{H}_{1}} d w(\mathbf{x}) \leq C_{1} \lambda
$$

Let

$$
\begin{aligned}
& f(\mathbf{x})=\mathbf{g}(\mathbf{x})+\sum_{Q_{j} \in \mathcal{Q}_{\lambda}}\left(f(\mathbf{x})-f_{Q_{j}}\right) \chi_{Q_{j}}(\mathbf{x})=\mathbf{g}(\mathbf{x})+\sum_{Q_{j} \in \mathcal{Q}_{\lambda}} \mathbf{b}_{j}(\mathbf{x})=\mathbf{g}(\mathbf{x})+\mathbf{b}(\mathbf{x}), \\
& f_{Q_{j}}=\frac{1}{w\left(Q_{j}\right)} \int_{Q_{j}} f(\mathbf{x}) d w(\mathbf{x}),
\end{aligned}
$$

be the Calderón-Zygmund decomposition at the level $\lambda$. Let $Q_{j}^{*}$ be the cube which has the same center $\mathbf{y}_{j}$ as $Q_{j}$ but whose diameter is expanded by the factor 2 . Set $\Omega=\bigcup_{Q_{j} \in \mathcal{Q}_{\lambda}} \mathcal{O}\left(Q_{j}^{*}\right)$, where, for a Lebesgue measurable set $U \subset \mathbb{R}^{N}$, we denote $\mathcal{O}(U)=$ $\{\sigma(\mathbf{x}): \mathbf{x} \in U, \sigma \in G\}$. Then by (2.5) and (3.3), we have

$$
w(\Omega) \leq \sum_{Q_{j} \in \mathcal{Q}_{\lambda}} w\left(\mathcal{O}\left(Q_{j}^{*}\right)\right) \leq C \sum_{Q_{j} \in \mathcal{Q}_{\lambda}} w\left(Q_{j}\right) \leq C \lambda^{-1}\|f\|_{L^{1}\left(d w, \mathcal{H}_{1}\right)}
$$

Clearly,

$\left\{\mathbf{x} \in \Omega^{c}:\|\mathcal{K} f(\mathbf{x})\|_{\mathcal{H}_{2}}>\lambda\right\} \subseteq\left\{\mathbf{x} \in \Omega^{c}:\|\mathcal{K} \mathbf{g}(\mathbf{x})\|_{\mathcal{H}_{2}}>\lambda / 2\right\} \cup\left\{\mathbf{x} \in \Omega^{c}:\|\mathcal{K} \mathbf{b}(\mathbf{x})\|_{\mathcal{H}_{2}}>\lambda / 2\right\}$.

By the boundendess of $\mathcal{K}$ from $L^{2}\left(d w, \mathcal{H}_{1}\right)$ to $L^{2}\left(d w, \mathcal{H}_{2}\right)$, we get

$$
w\left(\left\{\mathbf{x} \in \Omega^{c}:\|\mathcal{K} \mathbf{g}(\mathbf{x})\|_{\mathcal{H}_{2}}>\lambda / 2\right\}\right) \leq C \frac{\|\mathbf{g}\|_{L^{2}\left(d w, \mathcal{H}_{1}\right)}^{2}}{\lambda^{2}} \leq C \frac{\lambda\|f\|_{L^{1}\left(d w, \mathcal{H}_{1}\right)}}{\lambda^{2}}
$$


In order to estimate the measure of the second term we recall that $\int_{\mathbb{R}^{N}} \mathbf{b}_{j} d w=0$ and write

$$
\begin{aligned}
& w\left(\left\{\mathbf{x} \in \Omega^{c}:\|\mathcal{K} \mathbf{b}(\mathbf{x})\|_{\mathcal{H}_{2}}>\lambda / 2\right\}\right) \leq C \lambda^{-1}\left\|\chi_{\Omega^{c}} \mathcal{K} \mathbf{b}\right\|_{L^{1}\left(d w, \mathcal{H}_{2}\right)} \\
& =C \lambda^{-1} \int_{\Omega^{c}}\left\|\sum_{Q_{j} \in \mathcal{Q}_{\lambda}} \int_{\mathbb{R}^{N}} \mathcal{K}(\mathbf{x}, \mathbf{y}) \mathbf{b}_{j}(\mathbf{y}) d w(\mathbf{y})\right\|_{\mathcal{H}_{2}} d w(\mathbf{x}) \\
& =C \int_{\Omega^{c}}\left\|\sum_{Q_{j} \in \mathcal{Q}_{\lambda}} \int_{\mathbb{R}^{N}}\left[\mathcal{K}(\mathbf{x}, \mathbf{y})-\mathcal{K}\left(\mathbf{x}, \mathbf{y}_{j}\right)\right] \mathbf{b}_{j}(\mathbf{y}) d w(\mathbf{y})\right\|_{\mathcal{H}_{2}} d w(\mathbf{x}) \\
& \leq C \sum_{Q_{j} \in \mathcal{Q}_{\lambda}} \int_{\Omega^{c}} \int_{Q_{j}}\left\|\mathcal{K}(\mathbf{x}, \mathbf{y})-\mathcal{K}\left(\mathbf{x}, \mathbf{y}_{j}\right)\right\|_{\mathcal{L}\left(\mathcal{H}_{1}, \mathcal{H}_{2}\right)}\left\|\mathbf{b}_{j}(\mathbf{y})\right\|_{\mathcal{H}_{1}} d w(\mathbf{y}) d w(\mathbf{x}) \\
& \leq \sum_{Q_{j} \in \mathcal{Q}_{\lambda}} \int_{Q_{j}}\left\|b_{j}(\mathbf{y})\right\|_{\mathcal{H}_{1}} \int_{\left(\mathcal{O}\left(Q_{j}^{*}\right)\right)^{c}} \frac{C}{V(\mathbf{x}, \mathbf{y}, d(\mathbf{x}, \mathbf{y}))}\left(\frac{\left\|\mathbf{y}-\mathbf{y}_{j}\right\|}{d(\mathbf{x}, \mathbf{y})}\right)^{\delta^{\prime}} d w(\mathbf{x}) d w(\mathbf{y}) .
\end{aligned}
$$

Clearly, there is a constant $C>0$ such that for all $\mathbf{y} \in Q_{j} \in \mathcal{Q}_{\lambda}$ we have

$$
\int_{\left(\mathcal{O}\left(Q_{j}^{*}\right)\right)^{c}} \frac{1}{V(\mathbf{x}, \mathbf{y}, d(\mathbf{x}, \mathbf{y}))}\left(\frac{\left\|\mathbf{y}-\mathbf{y}_{j}^{\prime}\right\|}{d(\mathbf{x}, \mathbf{y})}\right)^{\delta^{\prime}} d w(\mathbf{x}) \leq C
$$

so, by (3.6), we get

$$
w\left(\left\{\mathbf{x} \in \Omega^{c}:\|\mathcal{K} \mathbf{b}(\mathbf{x})\|_{\mathcal{H}_{2}}>\lambda / 2\right\}\right) \leq \frac{C}{\lambda} \sum_{Q_{j} \in \mathcal{Q}_{\lambda}} \int_{\mathbb{R}^{N}}\left\|\mathbf{b}_{j}(\mathbf{y})\right\|_{\mathcal{H}_{1}} d w(\mathbf{y}) \leq \frac{C}{\lambda}\|f\|_{L^{1}\left(d w, \mathcal{H}_{1}\right)} .
$$

By (3.5) and (3.7) we obtain that $\mathcal{K}$ is of weak type $(1,1)$. Thanks to the vector-valued version of the Marcinkiewicz interpolation theorem (see e.g. [12, Exercise 5.5.3]) we obtain the claim for $1<p \leq 2$.

To prove the bounds for $2<p<\infty$, we apply the well-known duality argument. Observe that $\mathcal{K}^{*}$ is a bounded operator from the Hilbert space $L^{2}\left(d w, \mathcal{H}_{2}\right)$ to the Hilbert space $L^{2}\left(d w, \mathcal{H}_{1}\right)$ with the associated kernel $\mathcal{K}^{*}(\mathbf{x}, \mathbf{y})=\mathcal{K}(\mathbf{y}, \mathbf{x})^{*} \in \mathcal{L}\left(\mathcal{H}_{2}, \mathcal{H}_{1}\right)$. Hence, $\left\|\mathcal{K}^{*}(\mathbf{x}, \mathbf{y})\right\|_{\mathcal{L}\left(\mathcal{H}_{2}, \mathcal{H}_{1}\right)}=\|\mathcal{K}(\mathbf{y}, \mathbf{x})\|_{\mathcal{L}\left(\mathcal{H}_{1}, \mathcal{H}_{2}\right)}<\infty$ for $d(\mathbf{x}, \mathbf{y})>0$ and, if $2\left\|\mathbf{y}-\mathbf{y}^{\prime}\right\| \leq d(\mathbf{x}, \mathbf{y})$, then, by (3.2),

$$
\begin{aligned}
\left\|\mathcal{K}^{*}(\mathbf{x}, \mathbf{y})-\mathcal{K}^{*}\left(\mathbf{x}, \mathbf{y}^{\prime}\right)\right\|_{\mathcal{L}\left(\mathcal{H}_{2}, \mathcal{H}_{1}\right)} & =\left\|\mathcal{K}(\mathbf{y}, \mathbf{x})-\mathcal{K}\left(\mathbf{y}^{\prime}, \mathbf{x}\right)\right\|_{\mathcal{L}\left(\mathcal{H}_{1}, \mathcal{H}_{2}\right)} \\
& \leq \frac{C}{V(\mathbf{x}, \mathbf{y}, d(\mathbf{x}, \mathbf{y}))}\left(\frac{\left\|\mathbf{y}-\mathbf{y}^{\prime}\right\|}{d(\mathbf{x}, \mathbf{y})}\right)^{\delta^{\prime}}
\end{aligned}
$$

Let $\frac{1}{p}+\frac{1}{p^{\prime}}=1$. Note that $1<p^{\prime}<2$. Consequently, from the first part of the proof we conclude that there is a constant $C_{p^{\prime}}>0$ such that for all $g \in L^{p^{\prime}}\left(d w, \mathcal{H}_{2}\right)$ we have

$$
\left\|\mathcal{K}^{*} g\right\|_{L^{p^{\prime}}\left(d w, \mathcal{H}_{1}\right)} \leq C_{p^{\prime}}\|g\|_{L^{p^{\prime}\left(d w, \mathcal{H}_{2}\right)}} .
$$


Now, for $f \in L^{p}\left(d w, \mathcal{H}_{1}\right) \cap L^{2}\left(d w, \mathcal{H}_{1}\right), 2<p<\infty$, we write

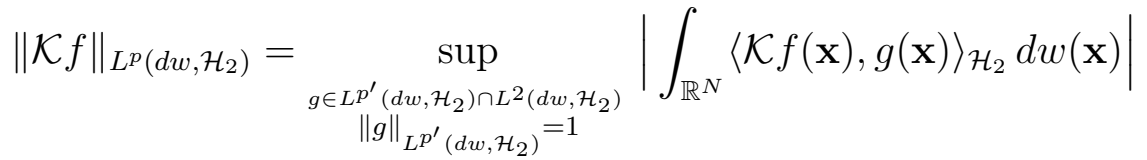

$$
\begin{aligned}
& =\sup _{\substack{g \in L^{p^{\prime}}\left(d w, \mathcal{H}_{2}\right) \cap L^{2}\left(d w, \mathcal{H}_{2}\right) \\
\|g\|_{L^{p^{\prime}}\left(d w, \mathcal{H}_{2}\right)}=1}}\left|\int_{\mathbb{R}^{N}}\left\langle f(\mathbf{x}), \mathcal{K}^{*} g(\mathbf{x})\right\rangle_{\mathcal{H}_{1}} d w(\mathbf{x})\right| \\
& \leq \sup _{\substack{g \in L^{p^{\prime}}\left(d w, \mathcal{H}_{2}\right) \cap L^{2}\left(d w, \mathcal{H}_{2}\right) \\
\|g\|_{L^{p^{\prime}}\left(d w, \mathcal{H}_{2}\right)}=1}} \int_{\mathbb{R}^{N}}\|f(\mathbf{x})\|_{\mathcal{H}_{1}}\left\|\mathcal{K}^{*} g(\mathbf{x})\right\|_{\mathcal{H}_{1}} d w(\mathbf{x})
\end{aligned}
$$

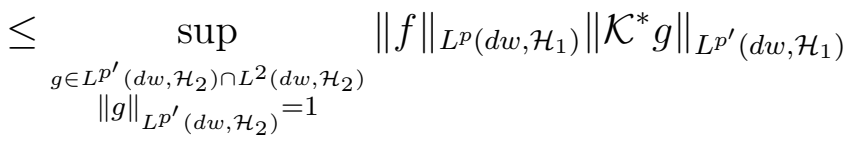

$$
\begin{aligned}
& \leq C_{p^{\prime}}\|f\|_{L^{p}\left(d w, \mathcal{H}_{1}\right)},
\end{aligned}
$$

where in the last inequality we have used (3.9).

3.2. Vector valued approach to square functions. For further applications we shall use $\mathcal{H}_{1}=\mathbb{C}$ and $\mathcal{H}_{2}=L^{2}\left((0, \infty), \frac{d t}{t}\right)$. Let $K(t, \mathbf{x}, \mathbf{y})$ be a measurable function on $(0, \infty) \times \mathbb{R}^{N} \times \mathbb{R}^{N}$ such that for certain $M^{\prime}>\mathbf{N}$ one has

$$
|K(t, \mathbf{x}, \mathbf{y})| \leq C \mathcal{V}(\mathbf{x}, \mathbf{y}, t)^{-1}\left(1+\frac{d(\mathbf{x}, \mathbf{y})}{t}\right)^{-M^{\prime}} .
$$

We additionally assume that there are constants $\delta>0$ and $C>0$ such that for all $\mathbf{x}, \mathbf{y}, \mathbf{y}^{\prime} \in \mathbb{R}^{N}$ and $t>0$, if $\left\|\mathbf{y}-\mathbf{y}^{\prime}\right\| \leq t$, then

$$
\begin{aligned}
& \left|K(t, \mathbf{x}, \mathbf{y})-K\left(t, \mathbf{x}, \mathbf{y}^{\prime}\right)\right| \leq C\left(\frac{\left\|\mathbf{y}-\mathbf{y}^{\prime}\right\|}{t}\right)^{\delta} \mathcal{V}(\mathbf{x}, \mathbf{y}, t)^{-1}\left(1+\frac{d(\mathbf{x}, \mathbf{y})}{t}\right)^{-M^{\prime}-\delta} \\
& \left|K(t, \mathbf{y}, \mathbf{x})-K\left(t, \mathbf{y}^{\prime}, \mathbf{x}\right)\right| \leq C\left(\frac{\left\|\mathbf{y}-\mathbf{y}^{\prime}\right\|}{t}\right)^{\delta} \mathcal{V}(\mathbf{x}, \mathbf{y}, t)^{-1}\left(1+\frac{d(\mathbf{x}, \mathbf{y})}{t}\right)^{-M^{\prime}-\delta} .
\end{aligned}
$$

Let

$$
K_{t} f(\mathbf{x})=\int_{\mathbb{R}^{N}} K(t, \mathbf{x}, \mathbf{y}) f(\mathbf{y}) d w(\mathbf{y})
$$

We define the square function $S_{K}$ associated with the kernel $K(t, \mathbf{x}, \mathbf{y})$ by

$$
S_{K}(f)(\mathbf{x})=\left(\int_{0}^{\infty}\left|K_{t} f(\mathbf{x})\right|^{2} \frac{d t}{t}\right)^{1 / 2} .
$$

Theorem 3.2. Assume that an integral kernel $K(t, \mathbf{x}, \mathbf{y})$ satisfies (3.11)-(3.13) with certain $M^{\prime}>\mathbf{N}$ and $\delta>0$, and the associated square function $S_{K}$ is bounded from $L^{2}(d w)$ into itself. Then for every $1<p<\infty$ there is a constant $C_{p}>0$ such that

$$
\left\|S_{K}(f)\right\|_{L^{p}(d w)} \leq C_{p}\|f\|_{L^{p}(d w)} \quad \text { for all } f \in L^{p}(d w) .
$$

Proof. In order to prove Theorem 3.2 we note that the boundedness of the square function $S_{K}$ on $L^{p}(d w)$-spaces is equivalent to the boundedness of the operator $\mathcal{K}$ from $L^{p}\left(d w, \mathcal{H}_{1}\right)$ to $L^{p}\left(d w, \mathcal{H}_{2}\right), \mathcal{H}_{1}=\mathbb{C}, \mathcal{H}_{2}=L^{2}\left((0, \infty), \frac{d t}{t}\right)$, where $\mathcal{K}$ is defined by

$$
\mathcal{K} f(\mathbf{x})(t)=\int_{\mathbb{R}^{N}} K_{t}(\mathbf{x}, \mathbf{y}) f(\mathbf{y}) d w(\mathbf{y}) .
$$


Recall that the boundedness of $\mathcal{K}$ from $L^{2}\left(d w, \mathcal{H}_{1}\right)$ to $L^{2}\left(d w, \mathcal{H}_{2}\right)$ is guaranteed by one of the assumptions. Therefore, to finish the proof, it suffices to check that the associated kernel $\mathcal{K}(\mathbf{x}, \mathbf{y})=K(t, \mathbf{x}, \mathbf{y})$ belongs to $\mathcal{L}\left(\mathcal{H}_{1}, \mathcal{H}_{2}\right)$ for $d(\mathbf{x}, \mathbf{y})>0$ and satisfies (3.1) and (3.2) with $\delta^{\prime}=\min \left(\delta, M^{\prime}-\mathbf{N}\right)>0$, and then apply Proposition 3.1. To verify the first requirement we shall prove that there is a constant $C>0$ such that for $d(\mathbf{x}, \mathbf{y})>0$ one has

$$
\|\mathcal{K}(\mathbf{x}, \mathbf{y})\|_{\mathcal{L}\left(\mathcal{H}_{1}, \mathcal{H}_{2}\right)} \leq C w(B(\mathbf{x}, d(\mathbf{x}, \mathbf{y})))^{-1} .
$$

By (3.11),

$$
\begin{aligned}
\|\mathcal{K}(\mathbf{x}, \mathbf{y})\|_{\mathcal{L}\left(\mathcal{H}_{1}, \mathcal{H}_{2}\right)} & \leq C^{2} \int_{0}^{\infty} \frac{1}{\mathcal{V}(\mathbf{x}, \mathbf{y}, t)^{2}}\left(1+\frac{d(\mathbf{x}, \mathbf{y})}{t}\right)^{-2 M^{\prime}} \frac{d t}{t} \\
& =C^{2} \int_{0}^{\infty} \frac{1}{\mathcal{V}(\mathbf{x}, \mathbf{y}, t d(\mathbf{x}, \mathbf{y}))^{2}}\left(1+\frac{1}{t}\right)^{-2 M^{\prime}} \frac{d t}{t} \\
& =\frac{C^{2}}{\mathcal{V}(\mathbf{x}, \mathbf{y}, d(\mathbf{x}, \mathbf{y}))^{2}} \int_{0}^{\infty} \frac{\mathcal{V}(\mathbf{x}, \mathbf{y}, d(\mathbf{x}, \mathbf{y})))^{2}}{\mathcal{V}(\mathbf{x}, \mathbf{y}, t d(\mathbf{x}, \mathbf{y}))^{2}}\left(1+\frac{1}{t}\right)^{-2 M^{\prime}} \frac{d t}{t}
\end{aligned}
$$

By (2.19) we have $\mathcal{V}(\mathbf{x}, \mathbf{y}, d(\mathbf{x}, \mathbf{y})) \sim w(B(\mathbf{x}, d(\mathbf{x}, \mathbf{y})))$. Applying the second inequality of (2.6) for $0<t<1$ and the first one for $t \geq 1$ we get the claim.

We now turn to verify (3.1). Note that $d(\mathbf{x}, \mathbf{y}) \sim d\left(\mathbf{x}, \mathbf{y}^{\prime}\right)$ for $d(\mathbf{x}, \mathbf{y})>2\left\|\mathbf{y}-\mathbf{y}^{\prime}\right\|$. Hence, by (3.11) and (3.12) we have

$$
\begin{aligned}
& \left\|\mathcal{K}(\mathbf{x}, \mathbf{y})-\mathcal{K}\left(\mathbf{x}, \mathbf{y}^{\prime}\right)\right\|_{\mathcal{L}\left(\mathcal{H}_{1}, \mathcal{H}_{2}\right)}^{2} \leq C \int_{0}^{\left\|\mathbf{y}-\mathbf{y}^{\prime}\right\|} \mathcal{V}(\mathbf{x}, \mathbf{y}, t)^{-2}\left(1+\frac{d(\mathbf{x}, \mathbf{y})}{t}\right)^{-2 M^{\prime}} \frac{d t}{t} \\
& \quad+C \int_{\left\|\mathbf{y}-\mathbf{y}^{\prime}\right\|}^{\infty} \mathcal{V}(\mathbf{x}, \mathbf{y}, t)^{-2}\left(\frac{\left\|\mathbf{y}-\mathbf{y}^{\prime}\right\|}{t}\right)^{2 \delta}\left(1+\frac{d(\mathbf{x}, \mathbf{y})}{t}\right)^{-2 M^{\prime}-2 \delta} \frac{d t}{t} \\
& \quad \leq C \int_{0}^{\left\|\mathbf{y}-\mathbf{y}^{\prime}\right\| / d(\mathbf{x}, \mathbf{y})} \mathcal{V}(\mathbf{x}, \mathbf{y}, t d(\mathbf{x}, \mathbf{y}))^{-2}\left(1+\frac{1}{t}\right)^{-2 M^{\prime}} \frac{d t}{t} \\
& \quad+C \int_{\left\|\mathbf{y}-\mathbf{y}^{\prime}\right\| / d(\mathbf{x}, \mathbf{y})}^{\infty}\left(\frac{\left\|\mathbf{y}-\mathbf{y}^{\prime}\right\|}{t d(\mathbf{x}, \mathbf{y})}\right)^{2 \delta} \mathcal{V}(\mathbf{x}, \mathbf{y}, t d(\mathbf{x}, \mathbf{y}))^{-2}\left(1+\frac{1}{t}\right)^{-2 M^{\prime}-2 \delta} \frac{d t}{t} \\
& \leq \frac{C}{\mathcal{V}(\mathbf{x}, \mathbf{y}, d(\mathbf{x}, \mathbf{y}))^{2}} \int_{0}^{\left\|\mathbf{y}-\mathbf{y}^{\prime}\right\| / d(\mathbf{x}, \mathbf{y})} \frac{\mathcal{V}(\mathbf{x}, d(\mathbf{x}, \mathbf{y}))^{2}}{\mathcal{V}(\mathbf{x}, \mathbf{y}, t d(\mathbf{x}, \mathbf{y})))^{2}} t^{2 M^{\prime}} \frac{d t}{t} \\
& \quad+\frac{C}{\mathcal{V}(\mathbf{x}, \mathbf{y}, d(\mathbf{x}, \mathbf{y}))^{2}} \int_{0}^{\infty}\left(\frac{\left\|\mathbf{y}-\mathbf{y}^{\prime}\right\|}{d(\mathbf{x}, \mathbf{y})}\right)^{2 \delta} t^{-2 \delta} \frac{\mathcal{V}(\mathbf{x}, \mathbf{y}, d(\mathbf{x}, \mathbf{y}))^{2}}{\mathcal{V}(\mathbf{x}, \mathbf{y}, t d(\mathbf{x}, \mathbf{y}))^{2}}\left(1+\frac{1}{t}\right)^{-2 M^{\prime}-2 \delta} \frac{d t}{t}
\end{aligned}
$$

Applying (2.6) together with (2.19), we get

$$
\begin{aligned}
\| \mathcal{K}(\mathbf{x}, \mathbf{y}) & -\mathcal{K}\left(\mathbf{x}, \mathbf{y}^{\prime}\right) \|_{\mathcal{L}\left(\mathcal{H}_{1}, \mathcal{H}_{2}\right)} \leq \frac{C}{w(B(\mathbf{x}, d(\mathbf{x}, \mathbf{y})))^{2}} \int_{0}^{\left\|\mathbf{y}-\mathbf{y}^{\prime}\right\| / d(\mathbf{x}, \mathbf{y})} t^{2 M^{\prime}-2 \mathbf{N}} \frac{d t}{t} \\
& +\frac{C}{w(B(\mathbf{x}, d(\mathbf{x}, \mathbf{y})))^{2}} \int_{0}^{\infty}\left(\frac{\left\|\mathbf{y}-\mathbf{y}^{\prime}\right\|}{d(\mathbf{x}, \mathbf{y})}\right)^{2 \delta} t^{-2 \delta}\left(t^{-\mathbf{N}}+t^{-N}\right)^{2}\left(1+\frac{1}{t}\right)^{-2 M^{\prime}-2 \delta} \frac{d t}{t} \\
& \leq \frac{C}{w(B(\mathbf{x}, d(\mathbf{x}, \mathbf{y})))^{2}}\left(\frac{\left\|\mathbf{y}-\mathbf{y}^{\prime}\right\|^{2 M^{\prime}-2 \mathbf{N}}}{d(\mathbf{x}, \mathbf{y})^{2 M^{\prime}-2 \mathbf{N}}}+\frac{\left\|\mathbf{y}-\mathbf{y}^{\prime}\right\|^{2 \delta}}{d(\mathbf{x}, \mathbf{y})^{2 \delta}}\right) .
\end{aligned}
$$

The proof of (3.2) is identical to that of (3.1) and uses (3.13). 


\section{Bessel potentials}

For a real number $s>0$ we set

$$
J^{\{s\}}(\mathbf{x})=\mathcal{F}^{-1}\left(\left(1+\|\cdot\|^{2}\right)^{-s / 2}\right)(\mathbf{x}) .
$$

By the gamma function identity we have

$$
\left(1+\|\xi\|^{2}\right)^{-s / 2}=\Gamma\left(\frac{s}{2}\right)^{-1} \int_{0}^{\infty} e^{-t} e^{-t\|\xi\|^{2}} t^{s / 2} \frac{d t}{t},
$$

which leads us to

$$
J^{\{s\}}(\mathbf{x})=\Gamma\left(\frac{s}{2}\right)^{-1} \int_{0}^{\infty} e^{-t} h_{t}(\mathbf{x}) t^{s / 2} \frac{d t}{t}
$$

(see $(2.17))$. The function $J^{\{s\}}$ is radial, positive and belongs $L^{1}(d w)$. Moreover, by $(2.14)$, for all $s_{1}, s_{2}>0$ we have

$$
J^{\left\{s_{1}\right\}} * J^{\left\{s_{2}\right\}}=c_{k} J^{\left\{s_{1}+s_{2}\right\}} .
$$

4.1. Pointwise estimates for the Bessel integral kernel. The following proposition is an easy consequence of (4.1) and (2.17).

Proposition 4.1. Let $M>0$. There is a constant $C=C_{s, M}>0$ such that

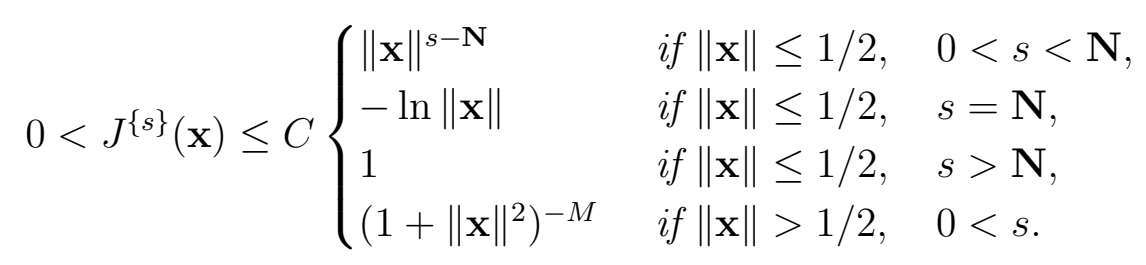

Proposition 4.2. Assume that $M \geq 0$ and $|f(\mathbf{x})| \leq(1+\|\mathbf{x}\|)^{-M-\mathbf{N}}$ for all $\mathbf{x} \in \mathbb{R}^{N}$. Let $s>\frac{\mathbf{N}}{2}$. Then there is a constant $C>0$ such that for all $\mathbf{x}, \mathbf{y} \in \mathbb{R}^{N}$ we have

$$
\left|\left(f * J^{\{s\}} * J^{\{s\}}\right)(\mathbf{x}, \mathbf{y})\right| \leq C \mathcal{V}(\mathbf{x}, \mathbf{y}, 1)^{-1}(1+d(\mathbf{x}, \mathbf{y}))^{-M} .
$$

Proof. Let $U_{j}=B\left(0,2^{j}\right) \backslash B\left(0,2^{j-1}\right)$ for $j \in \mathbb{Z}$. Set

$$
\phi_{j}(\mathbf{x})=J^{\{s\}}(\mathbf{x}) \chi_{U_{j}}(\mathbf{x}) .
$$

Let $V_{0}=B(0,1), V_{j}=U_{j}$ for $j>0$. For $j \in \mathbb{N}_{0}$ we set

$$
f_{j}(\mathbf{x})=f(\mathbf{x}) \chi_{V_{j}}(\mathbf{x})
$$

Thanks to Proposition 4.1, for every $D>0$ large enough, we have

$$
\left\|f_{j}\right\|_{L^{1}(d w)} \leq C w\left(B\left(0,2^{j}\right)\right) 2^{-j(M+\mathbf{N})}, \quad\left\|\phi_{\ell}\right\|_{L^{\infty}} \leq C_{D} \min \left(2^{-\ell D}, 2^{\ell(s-\mathbf{N})}\right)
$$

with $C, C_{D}>0$ independent of $j \in \mathbb{N}_{0}$ and $\ell \in \mathbb{Z}$. Then

$$
\left(f * J^{\{s\}} * J^{\{s\}}\right)(\mathbf{x}, \mathbf{y})=\sum_{j \in \mathbb{N}_{0}, \ell, m \in \mathbb{Z}}\left(f_{j} * \phi_{\ell} * \phi_{m}\right)(\mathbf{x}, \mathbf{y}) .
$$

Lemma 4.3 of [10] asserts that

$$
\left(\int_{\mathbb{R}^{N}} \phi_{m}(\mathbf{z}, \mathbf{y})^{2} d w(\mathbf{z})\right)^{1 / 2} \leq C C_{D} \frac{2^{m \mathbf{N}} \min \left(2^{-m D}, 2^{m(s-\mathbf{N})}\right)}{w\left(B\left(\mathbf{y}, 2^{m}\right)\right)^{1 / 2}} \leq C C_{D} \frac{\min \left(2^{m(\mathbf{N}-D)}, 2^{m(s-\mathbf{N} / 2)}\right)}{w(B(\mathbf{y}, 1))^{1 / 2}} .
$$

Inequality (4.8) of [10] asserts that

$$
\left(\int_{\mathbb{R}^{N}}\left|f_{j} * \phi_{\ell}(\mathbf{x}, \mathbf{z})\right|^{2} d w(\mathbf{z})\right)^{1 / 2} \leq C C_{D} \frac{\min \left(2^{\ell(\mathbf{N}-D)}, 2^{\ell(s-\mathbf{N} / 2)}\right)}{w(B(\mathbf{x}, 1))^{1 / 2}} 2^{-j M} .
$$


Hence, by the Cauchy-Schwarz inequality,

$$
\begin{aligned}
\left|\left(f_{j} * \phi_{\ell} * \phi_{m}\right)(\mathbf{x}, \mathbf{y})\right| & \leq C C_{D}^{2} \frac{\min \left(2^{\ell(\mathbf{N}-D)}, 2^{\ell(s-\mathbf{N} / 2)}\right)}{w(B(\mathbf{x}, 1))^{1 / 2}} \frac{\min \left(2^{m(\mathbf{N}-D)}, 2^{m(s-\mathbf{N} / 2)}\right)}{w(B(\mathbf{y}, 1))^{1 / 2}} 2^{-j M} \\
& =: s_{j, m, \ell}(\mathbf{x}, \mathbf{y}) .
\end{aligned}
$$

Observe that $\sum_{j \geq 0, m, \ell \in \mathbb{Z}} s_{j, m, \ell}(\mathbf{x}, \mathbf{y}) \leq C \mathcal{V}(\mathbf{x}, \mathbf{y}, 1)^{-1}$. Therefore, to finish the proof, we consider $d(\mathbf{x}, \mathbf{y}) \geq 1$. Recall that $\left(f_{j} * \phi_{\ell} * \phi_{m}\right)(\mathbf{x}, \mathbf{y})=0$ if $d(\mathbf{x}, \mathbf{y})>2^{j+\ell+m}$. Hence,

$$
\begin{aligned}
& \sum_{j \geq 0, m, \ell \in \mathbb{Z}}\left|\left(f_{j} * \phi_{\ell} * \phi_{m}\right)(\mathbf{x}, \mathbf{y})\right| \leq \sum_{m, \ell \in \mathbb{Z}} \sum_{j \geq 0: 2^{j} \geq 2^{-\ell-m} d(\mathbf{x}, \mathbf{y})} s_{j, m, \ell}(\mathbf{x}, \mathbf{y}) \\
\leq & C \sum_{m, \ell \in \mathbb{Z}} \frac{\min \left(2^{m(\mathbf{N}-D)}, 2^{m(s-\mathbf{N} / 2)}\right) \min \left(2^{\ell(\mathbf{N}-D)}, 2^{\ell(s-\mathbf{N} / 2)}\right)\left(d(\mathbf{x}, \mathbf{y}) 2^{-\ell-m}\right)^{-M}}{w(B(\mathbf{x}, 1))^{1 / 2} w(B(\mathbf{y}, 1))^{1 / 2}} \\
\leq & C \mathcal{V}(\mathbf{x}, \mathbf{y}, 1)^{-1} d(\mathbf{x}, \mathbf{y})^{-M}
\end{aligned}
$$

\section{2. $L^{1}$ estimates for Bessel potentials.}

Lemma 4.3. Let $M \geq 0, \kappa>3 \mathrm{~N} / 2+M$, and $\gamma \geq 0$. Suppose that a measurable function $f:(0, \infty) \times \mathbb{R}^{N} \times \mathbb{R}^{N} \rightarrow \mathbb{C}$ satisfies

$$
|f(t, \mathbf{x}, \mathbf{y})| \leq t^{-\gamma / 2} V(\mathbf{x}, \mathbf{y}, \sqrt{t})^{-1}\left(1+\frac{d(\mathbf{x}, \mathbf{y})}{\sqrt{t}}\right)^{-\kappa}
$$

for all $\mathbf{x}, \mathbf{y} \in \mathbb{R}^{N}$ and $t>0$. Then there is a constant $C=C_{M, \kappa, \gamma}>0$ such that for all $\mathbf{y} \in \mathbb{R}^{N}$ and $t>0$ we have

$$
\int_{\mathbb{R}^{N}} \frac{|f(t, \mathbf{x}, \mathbf{y})|}{w(B(\mathbf{x}, 1))^{1 / 2}}(1+d(\mathbf{x}, \mathbf{y}))^{M} d w(\mathbf{x}) \leq C t^{-\gamma / 2} \frac{(1+\sqrt{t})^{M+\mathbf{N} / 2}}{w(B(\mathbf{y}, 1))^{1 / 2}} .
$$

If additionally, for all $\mathbf{x}, \mathbf{y}, \mathbf{y}^{\prime} \in \mathbb{R}^{N}$ and $\left\|\mathbf{y}-\mathbf{y}^{\prime}\right\| \leq 1$ we have

$$
\left|f(t, \mathbf{x}, \mathbf{y})-f\left(t, \mathbf{x}, \mathbf{y}^{\prime}\right)\right| \leq \frac{\left\|\mathbf{y}-\mathbf{y}^{\prime}\right\|}{\sqrt{t}} t^{-\gamma / 2} V(\mathbf{x}, \mathbf{y}, \sqrt{t})^{-1}\left(1+\frac{d(\mathbf{x}, \mathbf{y})}{\sqrt{t}}\right)^{-\kappa}
$$

then for any $\delta \in[0,1]$ there is a constant $C=C_{M, \kappa, \gamma, \delta}>0$ such that for all $\mathbf{y}, \mathbf{y}^{\prime} \in \mathbb{R}^{N}$ such that $\left\|\mathbf{y}-\mathbf{y}^{\prime}\right\| \leq 1$ we have

$$
\begin{aligned}
& \int_{\mathbb{R}^{N}} \frac{\left|f(t, \mathbf{x}, \mathbf{y})-f\left(t, \mathbf{x}, \mathbf{y}^{\prime}\right)\right|}{w(B(\mathbf{x}, 1))^{1 / 2}}(1+d(\mathbf{x}, \mathbf{y}))^{M} d w(\mathbf{x}) \\
& \leq C t^{-\gamma / 2} \frac{\left\|\mathbf{y}-\mathbf{y}^{\prime}\right\|^{\delta}}{t^{\delta / 2}} \frac{(1+\sqrt{t})^{M+\mathbf{N} / 2}}{w(B(\mathbf{y}, 1))^{1 / 2}} .
\end{aligned}
$$

Proof. It follows from (2.6) and (2.19) that $w(B(\mathbf{x}, 1))^{-1 / 2} \leq C(B(\mathbf{y}, 1))^{-1 / 2}(1+d(\mathbf{x}, \mathbf{y}))^{\mathbf{N} / 2}$. We have

$$
\begin{aligned}
& \int_{\mathbb{R}^{N}} \frac{|f(t, \mathbf{x}, \mathbf{y})|}{w(B(\mathbf{x}, 1))^{1 / 2}}(1+d(\mathbf{x}, \mathbf{y}))^{M} d w(\mathbf{x}) \leq C \int_{\mathbb{R}^{N}} \frac{|f(t, \mathbf{x}, \mathbf{y})|}{w(B(\mathbf{y}, 1))^{1 / 2}}(1+d(\mathbf{x}, \mathbf{y}))^{M+\mathbf{N} / 2} d w(\mathbf{x}) \\
& \leq C \int_{\mathbb{R}^{N}} \frac{|f(t, \mathbf{x}, \mathbf{y})|}{w(B(\mathbf{y}, 1))^{1 / 2}}\left(1+\frac{d(\mathbf{x}, \mathbf{y})}{\sqrt{t}}\right)^{M+\mathbf{N} / 2}(1+\sqrt{t})^{M+\mathbf{N} / 2} d w(\mathbf{x}) \\
& \leq C t^{-\gamma / 2}(1+\sqrt{t})^{M+\mathbf{N} / 2} w(B(\mathbf{y}, 1))^{-1 / 2} .
\end{aligned}
$$

where in the last estimate we have used the assumption $\kappa>3 \mathbf{N} / 2+M$. 
In order to prove (4.7) we note that for all $\mathbf{x}, \mathbf{y}, \mathbf{y}^{\prime} \in \mathbb{R}^{N}$ such that $\left\|\mathbf{y}-\mathbf{y}^{\prime}\right\| \leq 1$ we have

$$
(1+d(\mathbf{x}, \mathbf{y}))^{M} \sim\left(1+d\left(\mathbf{x}, \mathbf{y}^{\prime}\right)\right)^{M} \quad \text { and } \quad w(B(\mathbf{y}, 1)) \sim w\left(B\left(\mathbf{y}^{\prime}, 1\right)\right) .
$$

Consequently, by (4.5),

$$
\int_{\mathbb{R}^{N}} \frac{\left|f\left(t, \mathbf{x}, \mathbf{y}^{\prime}\right)\right|}{w(B(\mathbf{x}, 1))^{1 / 2}}(1+d(\mathbf{x}, \mathbf{y}))^{M} d w(\mathbf{x}) \leq C t^{-\gamma / 2} \frac{(1+\sqrt{t})^{M+\mathbf{N} / 2}}{w(B(\mathbf{y}, 1))^{1 / 2}}
$$

Furthermore, if $0<\left\|\mathbf{y}-\mathbf{y}^{\prime}\right\| \leq 1$, then, by (4.5) applied to

$$
\widetilde{f}(t, \mathbf{x}, \mathbf{y})=\frac{1}{\left\|\mathbf{y}-\mathbf{y}^{\prime}\right\|}\left(f(t, \mathbf{x}, \mathbf{y})-f\left(t, \mathbf{x}, \mathbf{y}^{\prime}\right)\right)
$$

with $\widetilde{\gamma}=\gamma+1$, we get

$$
\begin{aligned}
& \int_{\mathbb{R}^{N}} w(B(\mathbf{x}, 1))^{-1 / 2}\left|f(t, \mathbf{x}, \mathbf{y})-f\left(t, \mathbf{x}, \mathbf{y}^{\prime}\right)\right|(1+d(\mathbf{x}, \mathbf{y}))^{M} d w(\mathbf{x}) \\
& \leq C t^{-\gamma / 2} \frac{\left\|\mathbf{y}-\mathbf{y}^{\prime}\right\|}{\sqrt{t}}(1+\sqrt{t})^{M+\mathbf{N} / 2} w(B(\mathbf{y}, 1))^{-1 / 2} .
\end{aligned}
$$

Now (4.7) is a consequence of (4.5), (4.8), and (4.9).

Proposition 4.4. Let $\delta \in[0,1], M \geq 0, \beta, \beta^{\prime} \in \mathbb{N}_{0}^{N}$ and $s>|\beta|+\left|\beta^{\prime}\right|$. There is a constant $C=C_{M, \beta, \beta^{\prime}, s}>0$ such that for all $\mathbf{y} \in \mathbb{R}^{N}$ we have

$$
\int_{\mathbb{R}^{N}} w(B(\mathbf{x}, 1))^{-1 / 2}\left|\partial_{\mathbf{x}}^{\beta} \partial_{\mathbf{y}}^{\beta^{\prime}} J^{\{s\}}(\mathbf{x}, \mathbf{y})\right|(1+d(\mathbf{x}, \mathbf{y}))^{M} d w(\mathbf{x}) \leq \frac{C}{w(B(\mathbf{y}, 1))^{1 / 2}} .
$$

Moreover, if we assume that $s>\delta+|\beta|+\left|\beta^{\prime}\right|$, then there is a constant $C=C_{M, \beta, \beta^{\prime}, s, \delta}$ such that for all $\mathbf{y}, \mathbf{y}^{\prime} \in \mathbb{R}^{N}$ such that $\left\|\mathbf{y}-\mathbf{y}^{\prime}\right\| \leq 1$ we have

$\int_{\mathbb{R}^{N}} w(B(\mathbf{x}, 1))^{-1 / 2}\left|\partial_{\mathbf{x}}^{\beta} \partial_{\mathbf{y}}^{\beta^{\prime}} J^{\{s\}}(\mathbf{x}, \mathbf{y})-\partial_{\mathbf{x}}^{\beta} \partial_{\mathbf{y}}^{\beta^{\prime}} J^{\{s\}}\left(\mathbf{x}, \mathbf{y}^{\prime}\right)\right|(1+d(\mathbf{x}, \mathbf{y}))^{M} d w(\mathbf{x}) \leq \frac{C\left\|\mathbf{y}-\mathbf{y}^{\prime}\right\|^{\delta}}{w(B(\mathbf{y}, 1))^{1 / 2}}$.

Proof. Note that if $s>\gamma$, then there is a constant $C=C_{M, s}>0$ such that

$$
\int_{0}^{\infty} e^{-t} t^{-\gamma / 2}\left(1+t^{\mathbf{N} / 4}\right)(1+\sqrt{t})^{M} t^{s / 2} \frac{d t}{t} \leq C
$$

On the other hand, by Theorem 2.2 there is a constant $C=C_{\beta, \beta^{\prime}}>0$ such that

$$
f(t, \mathbf{x}, \mathbf{y})=C^{-1} \partial_{\mathbf{x}}^{\beta} \partial_{\mathbf{y}}^{\beta^{\prime}} h_{t}(\mathbf{x}, \mathbf{y})
$$

satisfies (4.4) with $\gamma=|\beta|+\left|\beta^{\prime}\right|$ and $\kappa=2 \mathbf{N}+M$. Therefore, (4.10) is a consequence of Lemma 4.3, (4.12), and (4.1). Similarly, by Theorem 2.2 and mean value theorem, there is a constant $C>0$ such $f(t, \mathbf{x}, \mathbf{y})$ defined in (4.13) satisfies (4.6) with $\gamma=|\beta|+\left|\beta^{\prime}\right|$ and $\kappa=2 \mathrm{~N}+M$. Consequently, (4.11) follows by Lemma 4.3, (4.12), and (4.1).

Proposition 4.5. Assume that $M \geq 0$ and $|f(\mathbf{x})| \leq(1+\|\mathbf{x}\|)^{-M-\mathbf{N}}$ for all $\mathbf{x} \in \mathbb{R}^{N}$. Let $s>\frac{\mathbf{N}}{2}$ and $0<\delta<2 s-\mathbf{N}, 0<\delta \leq 1$. Then there is a constant $C>0$ such that for all $\mathbf{x}, \mathbf{y}, \mathbf{y}^{\prime} \in \mathbb{R}^{N},\left\|\mathbf{y}-\mathbf{y}^{\prime}\right\| \leq 1$ we have $\left|\left(f * J^{\{s\}} * J^{\{s\}}\right)(\mathbf{x}, \mathbf{y})-\left(f * J^{\{s\}} * J^{\{s\}}\right)\left(\mathbf{x}, \mathbf{y}^{\prime}\right)\right| \leq C \mathcal{V}(\mathbf{x}, \mathbf{y}, 1)^{-1}\left\|\mathbf{y}-\mathbf{y}^{\prime}\right\|^{\delta}(1+d(\mathbf{x}, \mathbf{y}))^{-M}$.

Proof. Take $s_{1}>\mathrm{N} / 2$ and $s_{2}>0$ such that $2 s=2 s_{1}+s_{2}, s_{2}>\delta$. Then

$$
f * J^{\{2 s\}}=c_{k}^{-1} f * J^{\left\{2 s_{1}\right\}} * J^{\left\{s_{2}\right\}} .
$$


By Proposition 4.2 , for all $\mathbf{x}, \mathbf{z} \in \mathbb{R}^{N}$ we have

$$
\left|\left(f * J^{\left\{s_{1}\right\}} * J^{\left\{s_{1}\right\}}\right)(\mathbf{x}, \mathbf{z})\right| \leq C \mathcal{V}(\mathbf{x}, \mathbf{z}, 1)^{-1}(1+d(\mathbf{x}, \mathbf{z}))^{-M} .
$$

Applying (4.11) we obtain

$$
\begin{aligned}
(1 & +d(\mathbf{x}, \mathbf{y}))^{M}\left|f * J^{\{2 s\}}(\mathbf{x}, \mathbf{y})-f * J^{\{2 s\}}\left(\mathbf{x}, \mathbf{y}^{\prime}\right)\right| \\
& \leq C \int_{\mathbb{R}^{N}}(1+d(\mathbf{x}, \mathbf{z}))^{M}(1+d(\mathbf{z}, \mathbf{y}))^{M}\left|f * J^{\left\{2 s_{1}\right\}}(\mathbf{x}, \mathbf{z})\right| \cdot\left|J^{\left\{s_{2}\right\}}(\mathbf{z}, \mathbf{y})-J^{\left\{s_{2}\right\}}\left(\mathbf{z}, \mathbf{y}^{\prime}\right)\right| d w(\mathbf{z}) \\
& \leq C \int_{\mathbb{R}^{N}} \mathcal{V}(\mathbf{x}, \mathbf{z}, 1)^{-1}(1+d(\mathbf{z}, \mathbf{y}))^{M}\left|J^{\left\{s_{2}\right\}}(\mathbf{z}, \mathbf{y})-J^{\left\{s_{2}\right\}}\left(\mathbf{z}, \mathbf{y}^{\prime}\right)\right| d w(\mathbf{z}) \\
& \leq C \mathcal{V}(\mathbf{x}, \mathbf{y}, 1)^{-1}\left\|\mathbf{y}-\mathbf{y}^{\prime}\right\|^{\delta} .
\end{aligned}
$$

\section{Estimates OF KERNELS}

Proposition 5.1. Let $s$ be a positive integer such that $2 s>\mathbf{N}$. Assume that a function $\varphi \in C^{2 s}\left(\mathbb{R}^{N}\right)$ satisfies

$$
\left|\partial^{\beta} \varphi(\mathbf{x})\right| \leq C_{\beta}(1+\|\mathbf{x}\|)^{-M-\mathbf{N}} \text { for }|\beta| \leq 2 s,
$$

for certain $M>0$. Then there is a constant $C>0$ which depends on $C_{\beta}, M$ and s, such that

$$
\left|\varphi_{t}(\mathbf{x}, \mathbf{y})\right| \leq C \mathcal{V}(\mathbf{x}, \mathbf{y}, t)^{-1}\left(1+\frac{d(\mathbf{x}, \mathbf{y})}{t}\right)^{-M} .
$$

Moreover, if $0<\delta \leq 1$ is such that $\delta<2 s-\mathbf{N}$, then there is a constant $C>0$ such that for $\left\|\mathbf{y}-\mathbf{y}^{\prime}\right\| \leq t$ one has

$$
\left|\varphi_{t}(\mathbf{x}, \mathbf{y})-\varphi_{t}\left(\mathbf{x}, \mathbf{y}^{\prime}\right)\right| \leq C \mathcal{V}(\mathbf{x}, \mathbf{y}, t)^{-1} \frac{\left\|\mathbf{y}-\mathbf{y}^{\prime}\right\|^{\delta}}{t^{\delta}}\left(1+\frac{d(\mathbf{x}, \mathbf{y})}{t}\right)^{-M} .
$$

If additionally $2 s>\mathbf{N}+1$, then

$$
\left|\partial_{j, \mathbf{x}} \varphi_{t}(\mathbf{x}, \mathbf{y})\right| \leq C \mathcal{V}(\mathbf{x}, \mathbf{y}, t)^{-1} t^{-1}\left(1+\frac{d(\mathbf{x}, \mathbf{y})}{t}\right)^{-M}
$$

Moreover, if $\delta>0$ is such that $0<\delta \leq 1, \delta<2 s-\mathbf{N}-1$, then there is $C>0$ such that for $\left\|\mathbf{y}-\mathbf{y}^{\prime}\right\| \leq t$, one has

$$
\left|\partial_{j, \mathbf{x}} \varphi_{t}(\mathbf{x}, \mathbf{y})-\partial_{j, \mathbf{x}} \varphi_{t}\left(\mathbf{x}, \mathbf{y}^{\prime}\right)\right| \leq C \mathcal{V}(\mathbf{x}, \mathbf{y}, t)^{-1} t^{-1} \frac{\left\|\mathbf{y}-\mathbf{y}^{\prime}\right\|^{\delta}}{t^{\delta}}\left(1+\frac{d(\mathbf{x}, \mathbf{y})}{t}\right)^{-M} .
$$

Similarly, if $\left\|\mathbf{x}-\mathbf{x}^{\prime}\right\| \leq t$, then

$$
\left|\partial_{j, \mathbf{x}} \varphi_{t}(\mathbf{x}, \mathbf{y})-\partial_{j, \mathbf{x}} \varphi_{t}\left(\mathbf{x}^{\prime}, \mathbf{y}\right)\right| \leq C \mathcal{V}(\mathbf{x}, \mathbf{y}, t)^{-1} t^{-1} \frac{\left\|\mathbf{x}-\mathbf{x}^{\prime}\right\|^{\delta}}{t^{\delta}}\left(1+\frac{d(\mathbf{x}, \mathbf{y})}{t}\right)^{-M} .
$$

Proof. It suffices to prove (5.1)-(5.5) for $t=1$ and then use scaling.

Let $f=\left(I-\Delta_{k}\right)^{s} \varphi$. Then $\varphi=c_{k}^{-1} f * J^{\{s\}} * J^{\{s\}}$. Applying Proposition 4.2 we obtain (5.1).

We now turn to prove (5.2). Fix $0<\delta \leq 1, \delta<2 s-\mathbf{N}$. Let $s_{1}>\mathbf{N} / 2$ and $s_{2}>\delta$ be such that $2 s=2 s_{1}+s_{2}$. Then $\varphi=c_{k}^{-1} f * J^{\left\{2 s_{1}\right\}} * J^{\left\{s_{2}\right\}}$. By Proposition 4.2,

$$
\left|f * J^{\left\{2 s_{1}\right\}}(\mathbf{x}, \mathbf{z})\right| \leq C \mathcal{V}(\mathbf{x}, \mathbf{z}, 1)^{-1}(1+d(\mathbf{x}, \mathbf{z}))^{-M} .
$$


Applying (5.6) we get

$$
\begin{aligned}
& \left|\varphi_{t}(\mathbf{x}, \mathbf{y})-\varphi_{t}\left(\mathbf{x}, \mathbf{y}^{\prime}\right)\right|(1+d(\mathbf{x}, \mathbf{y}))^{M} \\
& \quad \leq C \int_{\mathbb{R}^{N}}(1+d(\mathbf{x}, \mathbf{z}))^{M}\left|\left(f * J^{\left\{2 s_{1}\right\}}\right)(\mathbf{x}, \mathbf{z})\right|(1+d(\mathbf{z}, \mathbf{y}))^{M}\left|J^{\left\{s_{2}\right\}}(\mathbf{z}, \mathbf{y})-J^{\left\{s_{2}\right\}}\left(\mathbf{z}, \mathbf{y}^{\prime}\right)\right| d w(\mathbf{z}) \\
& \quad \leq C \int_{\mathbb{R}^{N}} \mathcal{V}(\mathbf{x}, \mathbf{z}, 1)^{-1}(1+d(\mathbf{z}, \mathbf{y}))^{M}\left|J^{\left\{s_{2}\right\}}(\mathbf{z}, \mathbf{y})-J^{\left\{s_{2}\right\}}\left(\mathbf{z}, \mathbf{y}^{\prime}\right)\right| d w(\mathbf{z}) \\
& \quad \leq C \mathcal{V}(\mathbf{x}, \mathbf{y}, 1)^{-1}\left\|\mathbf{y}-\mathbf{y}^{\prime}\right\|^{\delta},
\end{aligned}
$$

where in the last inequality we have used (4.11) with $\beta=\beta^{\prime}=\mathbf{0}$.

In order to prove (5.3)-(5.4), we fix $0<\delta<2 s-\mathbf{N}-1, \delta \leq 1$, and take $s_{1}$ and $s_{2}$ such that $2 s_{1}>\mathbf{N}+\delta, s_{2}>1,2 s=2 s_{1}+s_{2}$. We write $\varphi=c_{k}^{-1} J^{\left\{s_{2}\right\}} *\left(f * J^{\left\{2 s_{1}\right\}}\right)$, where $f=\left(I-\Delta_{k}\right)^{s} \varphi$. Then

$$
\partial_{j, \mathbf{x}} \phi(\mathbf{x}, \mathbf{y})=c_{k}^{-1} \int_{\mathbb{R}^{N}} \partial_{j, \mathbf{x}} J^{\left\{s_{2}\right\}}(\mathbf{x}, \mathbf{z})\left(f * J^{\left\{2 s_{1}\right\}}\right)(\mathbf{z}, \mathbf{y}) d w(\mathbf{z}) .
$$

Now, having (2.15) in mind, we use Propositions 4.2, 4.4, and 4.5, and proceed as in the proofs of (5.1) and (5.2) to obtain (5.3)-(5.4).

The proof of (5.5) is identical, however, this time, for fixed $0<\delta<2 s-\mathbf{N}-1$, we use the formula (5.7) with $2 s_{1}>\mathbf{N}, s_{2}>1+\delta, 2 s=2 s_{1}+s_{2}$.

For $\alpha \in R$, set

$$
K^{\{\alpha\}}(t, \mathbf{x}, \mathbf{y})=t \frac{\phi_{t}(\mathbf{x}, \mathbf{y})-\phi_{t}\left(\sigma_{\alpha}(\mathbf{x}), \mathbf{y}\right)}{\langle\alpha, \mathbf{x}\rangle} .
$$

Proposition 5.2. Let $s$ be a positive integer such that $2 s>\mathbf{N}+1$. Assume that $\phi \in C^{2 s}\left(\mathbb{R}^{N}\right)$ satisfies

$$
\left|\partial^{\beta} \phi(\mathbf{x})\right| \leq(1+\|\mathbf{x}\|)^{-M-\mathbf{N}} \text { for all }|\beta| \leq 2 s,
$$

for certain $M>\lfloor\mathbf{N}\rfloor+1$. Then there is a constant $C>0$ such that for all $\mathbf{x}, \mathbf{y} \in \mathbb{R}^{N}$ and $t>0$ we have

$$
\left|K^{\{\alpha\}}(t, \mathbf{x}, \mathbf{y})\right| \leq C \mathcal{V}(\mathbf{x}, \mathbf{y}, t)^{-1}\left(1+\frac{d(\mathbf{x}, \mathbf{y})}{t}\right)^{-M} .
$$

Moreover, there is a constant $C>0$ and $0<\delta \leq 1$ such that for all $\mathbf{x}, \mathbf{y}, \mathbf{y}^{\prime} \in \mathbb{R}^{N}$ and $\left\|\mathbf{y}-\mathbf{y}^{\prime}\right\| \leq t$ one has

$$
\begin{aligned}
& \left|K^{\{\alpha\}}(t, \mathbf{x}, \mathbf{y})-K^{\{\alpha\}}\left(t, \mathbf{x}, \mathbf{y}^{\prime}\right)\right| \leq C \frac{\left\|\mathbf{y}-\mathbf{y}^{\prime}\right\|^{\delta}}{t^{\delta}} \mathcal{V}(\mathbf{x}, \mathbf{y}, t)^{-1}\left(1+\frac{d(\mathbf{x}, \mathbf{y})}{t}\right)^{-M} \\
& \left|K^{\{\alpha\}}(t, \mathbf{y}, \mathbf{x})-K^{\{\alpha\}}\left(t, \mathbf{y}^{\prime}, \mathbf{x}\right)\right| \leq C \frac{\left\|\mathbf{y}-\mathbf{y}^{\prime}\right\|^{\delta}}{t^{\delta}} \mathcal{V}(\mathbf{x}, \mathbf{y}, t)^{-1}\left(1+\frac{d(\mathbf{x}, \mathbf{y})}{t}\right)^{-M}
\end{aligned}
$$

Proof. Recall that $\left\|\mathbf{x}-\sigma_{\alpha}(\mathbf{x})\right\|=\sqrt{2}|\langle\alpha, \mathbf{x}\rangle|$ (see (2.1)). So, if $|\langle\alpha, \mathbf{x}\rangle|<t$, then (5.10) follows from (5.2) (with $\delta=1$ ). Otherwise we apply (5.1) to obtain (5.10), because, by $(2.18), d(\mathbf{x}, \mathbf{y})=d\left(\sigma_{\alpha}(\mathbf{x}), \mathbf{y}\right)$.

In order to prove (5.11) take $s_{1}, s_{2}>0$ such that $2 s=2 s_{1}+s_{2}, 2 s_{1}>\mathbf{N}+1$. Fix $0<\delta<s_{2}$. Set

$$
\begin{aligned}
& f(\mathbf{x})=\left(I-\Delta_{k}\right)^{s} \phi(\mathbf{x}), \quad \phi^{\{1\}}=J^{\left\{2 s_{1}\right\}} * f \\
& K^{\{1, \alpha\}}(t, \mathbf{x}, \mathbf{z})=\frac{t}{\langle\alpha, \mathbf{x}\rangle}\left(\phi_{t}^{\{1\}}(\mathbf{x}, \mathbf{z})-\phi_{t}^{\{1\}}\left(\sigma_{\alpha}(\mathbf{x}), \mathbf{z}\right)\right) .
\end{aligned}
$$


By (4.2) and Propositions 4.5 and 4.2 we have

$$
\begin{aligned}
\left|K^{\{1, \alpha\}}(t, \mathbf{x}, \mathbf{z})\right| & \leq C \begin{cases}\frac{t}{|\langle\alpha, \mathbf{x}\rangle|} \frac{\left\|\mathbf{x}-\sigma_{\alpha}(\mathbf{x})\right\|}{t} \mathcal{V}(\mathbf{x}, \mathbf{z}, t)^{-1}\left(1+\frac{d(\mathbf{x}, \mathbf{z})}{t}\right)^{-M} & \text { if }\left\|\mathbf{x}-\sigma_{\alpha}(\mathbf{x})\right\| \leq t \\
\mathcal{V}(\mathbf{x}, \mathbf{z}, t)^{-1}\left(1+\frac{d(\mathbf{x}, \mathbf{z})}{t}\right)^{-M} & \text { if }\left\|\mathbf{x}-\sigma_{\alpha}(\mathbf{x})\right\|>t\end{cases} \\
& \leq C \mathcal{V}(\mathbf{x}, \mathbf{z}, t)^{-1}\left(1+\frac{d(\mathbf{x}, \mathbf{z})}{t}\right)^{-M} .
\end{aligned}
$$

Finally, for $\left\|\mathbf{y}-\mathbf{y}^{\prime}\right\|<t$, applying Proposition 4.4, we arrive to

$$
\begin{aligned}
& (1+d(\mathbf{x}, \mathbf{y}))^{M}\left|K^{\{\alpha\}}(t, \mathbf{x}, \mathbf{y})-K^{\{\alpha\}}\left(t, \mathbf{x}, \mathbf{y}^{\prime}\right)\right| \\
& \quad \leq C\left|\int(1+d(\mathbf{x}, \mathbf{z}))^{M}(1+d(\mathbf{z}, \mathbf{y}))^{M} K^{\{1, \alpha\}}(t, \mathbf{x}, \mathbf{z})\left(\left(J^{\left\{s_{2}\right\}}\right)_{t}(\mathbf{z}, \mathbf{y})-\left(J^{\left\{s_{2}\right\}}\right)_{t}\left(\mathbf{z}, \mathbf{y}^{\prime}\right)\right) d w(\mathbf{z})\right| \\
& \quad \leq C \frac{\left\|\mathbf{y}-\mathbf{y}^{\prime}\right\|^{\delta}}{t^{\delta}} \mathcal{V}(\mathbf{x}, \mathbf{y}, t)^{-1},
\end{aligned}
$$

which proves (5.11).

We now turn to prove (5.12). We may assume that $\left\|\mathbf{y}-\mathbf{y}^{\prime}\right\|<\frac{1}{8} t$, otherwise, for $t / 8 \leq\left\|\mathbf{y}-\mathbf{y}^{\prime}\right\| \leq t$, the inequality (5.12) is a consequence of (5.10). We consider two cases.

Case 1: $\left\|\mathbf{y}-\sigma_{\alpha}(\mathbf{y})\right\|>t / 2$. Then $\sqrt{2}|\langle\mathbf{y}, \alpha\rangle|=\left\|\mathbf{y}-\sigma_{\alpha}(\mathbf{y})\right\|>t / 2$ and $\sqrt{2}\left|\left\langle\mathbf{y}^{\prime}, \alpha\right\rangle\right|=$ $\left\|\mathbf{y}^{\prime}-\sigma_{\alpha}\left(\mathbf{y}^{\prime}\right)\right\|>t / 4$. So by $(5.2)($ with $\delta=1)$ we get

$$
\begin{aligned}
\left|K^{\{\alpha\}}(t, \mathbf{y}, \mathbf{x})-K^{\{\alpha\}}\left(t, \mathbf{y}^{\prime}, \mathbf{x}\right)\right| & \leq \frac{t}{\left|\left\langle\mathbf{y}^{\prime}, \alpha\right\rangle\right|}\left|\phi_{t}(\mathbf{y}, \mathbf{x})-\phi_{t}\left(\mathbf{y}^{\prime}, \mathbf{x}\right)\right| \\
& +\frac{t}{\left|\left\langle\mathbf{y}^{\prime}, \alpha\right\rangle\right|}\left|\phi_{t}\left(\sigma_{\alpha}(\mathbf{y}), \mathbf{x}\right)-\phi_{t}\left(\sigma_{\alpha}\left(\mathbf{y}^{\prime}\right), \mathbf{x}\right)\right| \\
& +\frac{t}{\left|\langle\mathbf{y}, \alpha\rangle\left\langle\mathbf{y}^{\prime}, \alpha\right\rangle\right|}\left|\left\langle\mathbf{y}-\mathbf{y}^{\prime}, \alpha\right\rangle\right|\left(\left|\phi_{t}(\mathbf{y}, \mathbf{x})\right|+\left|\phi_{t}\left(\sigma_{\alpha}(\mathbf{y}), \mathbf{x}\right)\right|\right) \\
& \leq C \frac{\left\|\mathbf{y}-\mathbf{y}^{\prime}\right\|}{t} \mathcal{V}(\mathbf{y}, \mathbf{x}, t)^{-1}\left(1+\frac{d(\mathbf{x}, \mathbf{y})}{t}\right)^{-M} .
\end{aligned}
$$

Case 2: $\left\|\mathbf{y}-\sigma_{\alpha}(\mathbf{y})\right\| \leq t / 2$. For $\tau \in[0,1]$ we set $\mathbf{y}(\tau)=\tau\left(\mathbf{y}-\sigma_{\alpha}(\mathbf{y})\right)+\sigma_{\alpha}(\mathbf{y})$. Note that

$$
K^{\{\alpha\}}(t, \mathbf{y}, \mathbf{x})=\frac{t}{\langle\alpha, \mathbf{y}\rangle} \int_{0}^{1} \frac{d}{d \tau}\left\{\phi_{t}(\mathbf{y}(\tau), \mathbf{x})\right\} d \tau=t \int_{0}^{1}\left\langle\nabla_{(1)}\left(\phi_{t}\right)(\mathbf{y}(\tau), \mathbf{x}), \alpha\right\rangle d \tau,
$$

where the symbol $\nabla_{(1)}$ denotes the gradient with respect to the first $N$-variables. Observe that $\left\|\mathbf{y}(\tau)-\left(\mathbf{y}^{\prime}\right)(\tau)\right\| \leq C\left\|\mathbf{y}-\mathbf{y}^{\prime}\right\| \leq C t$. Hence, by (5.5) combined with (5.13), we obtain that there is $0<\delta \leq 1$ such that

$$
\left|K^{\{\alpha\}}(t, \mathbf{y}, \mathbf{x})-K^{\{\alpha\}}\left(t, \mathbf{y}^{\prime}, \mathbf{x}\right)\right| \leq C_{M} \frac{\left\|\mathbf{y}-\mathbf{y}^{\prime}\right\|^{\delta}}{t^{\delta}} \int_{0}^{1} V(\mathbf{y}(\tau), \mathbf{x}, t)^{-1}\left(1+\frac{d(\mathbf{y}(\tau), \mathbf{x})}{t}\right)^{-M} d \tau
$$

The assumption $\left\|\mathbf{y}-\sigma_{\alpha}(\mathbf{y})\right\| \leq t / 2$ implies

$$
d(\mathbf{y}(\tau), \mathbf{x}) \geq d(\mathbf{y}, \mathbf{x})-d(\mathbf{y}(\tau), \mathbf{y}) \geq d(\mathbf{y}, \mathbf{x})-\|\mathbf{y}(\tau)-\mathbf{y}\| \geq d(\mathbf{y}, \mathbf{x})-\frac{1}{2} t .
$$

Moreover, $\mathcal{V}(\mathbf{y}(\tau), \mathbf{x}, t) \sim \mathcal{V}(\mathbf{y}, \mathbf{x}, t)$. So, from (5.14) and (5.15), we conclude (5.12).

Corollary 5.3. Under assumptions of Proposition 5.2 the integral kernel $t \nabla_{k, \mathbf{x}} \phi_{t}(\mathbf{x}, \mathbf{y})$ associated with the square function $S_{\nabla_{k}, \phi}$ satisfies the conditions (3.11)-(3.13). 
Proof. The corollary is consequence of (2.7), Propositions 5.1 and 5.2, it suffices to take $M^{\prime}>\mathbf{N}$ and $\delta>0$ (small enough) such that $M=M^{\prime}+\delta$.

\section{6. $L^{2}(d w)$-BOUNDS FOR SQUARE FUNCTIONS}

In this section we assume that $\phi \in C^{2 s}\left(\mathbb{R}^{N}\right)$, for certain $s$ being a positive integer such that $2 s>\mathbf{N}+1$ and satisfies

$$
\left|\partial^{\beta} \phi(\mathbf{x})\right| \leq(1+\|\mathbf{x}\|)^{-M-\mathbf{N}} \text { for all }|\beta| \leq 2 s
$$

for certain $M>\lfloor\mathbf{N}\rfloor+1$.

By straightforward calculations (see [11, Lemma 4.4] or [25, Lemma 3.1]) we have

$$
\Gamma(f, g)(\mathbf{x})=\langle\nabla f(\mathbf{x}), \nabla g(\mathbf{x})\rangle+\sum_{\alpha \in R} \frac{k(\alpha)}{2} \frac{\left.\left(f(\mathbf{x})-f\left(\sigma_{\alpha}(\mathbf{x})\right)\right)\left(\overline{g(\mathbf{x})-g\left(\sigma_{\alpha}(\mathbf{x})\right.}\right)\right)}{\langle\alpha, \mathbf{x}\rangle^{2}} .
$$

Observe that $\Gamma\left(\phi_{t} * f, \phi_{t} * f\right)(\mathbf{x})$ is the sum of non-negative functions. Using (1.1), (1.2), (2.8), and Plancherel's formula (2.12) together with (2.13) we get

$$
\begin{aligned}
\| \mathfrak{g}_{\Gamma, \phi}(f) & \|_{L^{2}(d w)}^{2} \\
& =\frac{1}{2} \int_{0}^{\infty} t^{2} \int_{\mathbb{R}^{N}} \Delta_{k}\left(\left(\phi_{t} * f\right)\left(\overline{\phi_{t} * f}\right)\right)-\phi_{t} * f \Delta_{k}\left(\overline{\phi_{t} * f}\right)-\overline{\phi_{t} * f} \Delta_{k}\left(\phi_{t} * f\right) d w \frac{d t}{t} \\
& =\int_{0}^{\infty} \int_{\mathbb{R}^{N}} t^{2}\left|\nabla_{k}\left(\phi_{t} * f\right)(\mathbf{x})\right|^{2} d w(\mathbf{x}) \frac{d t}{t} \\
& =\int_{0}^{\infty} \int_{\mathbb{R}^{N}} t^{2}\|\xi\|^{2}|\mathcal{F} \phi(t \xi)|^{2}|\mathcal{F} f(\xi)|^{2} d w(\xi) \frac{d t}{t} \\
& =\int_{\mathbb{R}^{N}} c_{\phi}(\xi)|\mathcal{F} f(\xi)|^{2} d w(\xi),
\end{aligned}
$$

where

$$
c_{\phi}(\xi)=\int_{0}^{\infty} t^{2}\|\xi\|^{2}|\mathcal{F} \phi(t \xi)|^{2} \frac{d t}{t} .
$$

Observe that thanks to (2.26) of Proposition 2.3 (with $\beta^{\prime}=0$ and $\ell=2$ ) the function $c_{\phi}$ is bounded and homogeneous of degree 0. Using the Plancherel identity (2.12), we obtain

$$
\left\|\mathfrak{g}_{\Gamma, \phi} f\right\|_{L^{2}(d w)} \leq C\|f\|_{L^{2}(d w)} .
$$

For $\alpha \in R$ let

$$
S_{K\{\alpha\}} f(\mathbf{x})=\left(\int_{0}^{\infty}\left|K_{t}^{\{\alpha\}} f(\mathbf{x})\right|^{2} \frac{d t}{t}\right)^{1 / 2},
$$

where $K_{t}^{\{\alpha\}}$ is defined by (5.8). By (6.2) we have

$$
\mathfrak{g}_{\Gamma, \phi}(f)(\mathbf{x})^{2}=S_{\nabla, \phi} f(\mathbf{x})^{2}+\sum_{\alpha \in R} \frac{k(\alpha)}{2} S_{K^{\{\alpha\}}} f(\mathbf{x})^{2} .
$$

Since

$$
t T_{j} \phi_{t} * f(\mathbf{x})-t \partial_{j} \phi_{t} * f(\mathbf{x})=\sum_{\alpha \in R} \frac{k(\alpha)}{2} \alpha_{j} K_{t}^{\{\alpha\}} f(\mathbf{x})
$$


we obtain the pointwise bounds

$$
\begin{gathered}
S_{\nabla, \phi} f(\mathbf{x})+S_{\nabla_{k}, \phi} f(\mathbf{x})+\sum_{\alpha \in R} \frac{k(\alpha)}{2} S_{K^{\{\alpha\}}} f(\mathbf{x}) \leq C \mathfrak{g}_{\Gamma, \phi} f(\mathbf{x}), \\
\mathfrak{g}_{\Gamma, \phi}(f)(\mathbf{x}) \leq C\left(S_{\nabla_{k}, \phi} f(\mathbf{x})+\sum_{\alpha \in R} \frac{k(\alpha)}{2} S_{K^{\{\alpha\}}} f(\mathbf{x})\right) .
\end{gathered}
$$

Consequently, by (6.5) and (6.7),

$$
\left\|S_{\nabla, \phi} f\right\|_{L^{2}(d w)}+\left\|S_{\nabla_{k}, \phi} f\right\|_{L^{2}(d w)}+\sum_{\alpha \in R} k(\alpha)\left\|S_{K\{\alpha\}} f\right\|_{L^{2}(d w)} \leq C\|f\|_{L^{2}(d w)} .
$$

Assume that $\psi \in C^{2 s}\left(\mathbb{R}^{N}\right)$ satisfying $(6.1)$ is such that $\int_{\mathbb{R}^{N}} \psi(\mathbf{x}) d w(\mathbf{x})=0$. Let

$$
\tilde{c}_{\psi}(\xi)=\int_{0}^{\infty}|\mathcal{F} \psi(t \xi)|^{2} \frac{d t}{t}
$$

From Proposition 2.3 we conclude that $\tilde{c}_{\psi}(\xi)$ is a bounded homogeneous of degree 0 function. It can be proved using the Dunkl transform and the Plancherel identity (cf. (6.3)) that

$$
\left\|S_{\psi}(f)\right\|_{L^{2}(d w)}^{2}=\int|\mathcal{F} f(\xi)|^{2} \tilde{c}_{\psi}(\xi) d w(\xi) \leq C\|f\|_{L^{2}(d w)}^{2} .
$$

We finish this section by writing the following easily proved identities for $f, g \in L^{2}(d w)$ (cf. (6.3) and (6.10)):

$$
\begin{gathered}
\int_{\mathbb{R}^{N}} \int_{0}^{\infty} t^{2} \Gamma\left(\phi_{t} * f, \phi_{t} * g\right)(\mathbf{x}) \frac{d t}{t} d w(\mathbf{x})=\int_{\mathbb{R}^{N}} \mathcal{F} f(\xi) \overline{\mathcal{F} g(\xi)} c_{\phi}(\xi) d w(\xi) \\
\int_{\mathbb{R}^{N}} \int_{0}^{\infty} t^{2}\left\langle\nabla_{k}\left(\phi_{t} * f\right)(\mathbf{x}), \nabla_{k}\left(\phi_{t} * g\right)(\mathbf{x})\right\rangle \frac{d t}{t} d w(\mathbf{x})=\int_{\mathbb{R}^{N}} \mathcal{F} f(\xi) \overline{\mathcal{F} g(\xi)} c_{\phi}(\xi) d w(\xi) \\
\int_{\mathbb{R}^{N}} \int_{0}^{\infty} \psi_{t} * f(\mathbf{x}) \overline{\psi_{t} * g(\mathbf{x})} \frac{d t}{t} d w(\mathbf{x})=\int_{\mathbb{R}^{N}} \mathcal{F} f(\xi) \overline{\mathcal{F} g(\xi)} \tilde{c}_{\psi}(\xi) d w(\xi)
\end{gathered}
$$

\section{Proofs of Theorems 1.1, 1.2, And Corollary 1.3}

We start by proving Theorem 1.1. To this end, by (6.7) and (6.8), it suffices to establish that for every $1<p<\infty$ and $\alpha \in R$ the square functions $S_{\nabla_{k}, \phi}, k(\alpha) S_{K\{\alpha\}}$, and $S_{\psi}$ are bounded on $L^{p}(d w)$. The $L^{2}(d w)$-bounds of the square functions are guaranteed by (6.9) and (6.10). To finish the proof of Theorem 1.1 it suffices to check that the associated kernels $t \nabla_{k, \mathbf{x}} \phi_{t}(\mathbf{x}, \mathbf{y}), k(\alpha) K^{\{\alpha\}}(t, \mathbf{x}, \mathbf{y})$, and $K_{\psi}(t, \mathbf{x}, \mathbf{y})=\psi_{t}(\mathbf{x}, \mathbf{y})$ satisfy (3.11)-(3.13) and then apply Theorem 3.2. But these are guaranteed by Corollary 5.3, Proposition 5.2, and Proposition 5.1.

We now turn to prove Theorem 1.2. We start by verifying (1.6). Proposition 2.3 implies that $\mathcal{F} \phi \in C^{\lfloor\mathbf{N}\rfloor+1}\left(\mathbb{R}^{N}\right)$ and

$$
\left|\partial^{\beta^{\prime}} \mathcal{F} \phi(\xi)\right| \leq C_{\beta^{\prime}}(1+\|\xi\|)^{-2 s} \quad \text { for }\left|\beta^{\prime}\right| \leq\lfloor\mathbf{N}\rfloor+1 .
$$

Thus it is easy to see that the function $c_{\phi}$ (defined by (6.4)) is $C^{\lfloor\mathbf{N}\rfloor+1}$ away from the origin and homogeneous of degree zero.

Recall that by our assumption, $\mathcal{F} \phi$ is not identically zero along any direction (see (1.5)). Hence, there is a constant $C>0$ such that $0<C^{-1} \leq c_{\phi}(\xi) \leq C$ for all $\xi \neq 0$. Now, Theorem 1.2 of [10] asserts that for every $1<q<\infty$, the Dunkl multiplier operator

$$
f \mapsto \mathcal{T}_{c_{\phi}} f:=\mathcal{F}^{-1}\left(c_{\phi}(\xi) \mathcal{F} f(\xi)\right),
$$


initially defined on $L^{q}(d w) \cap L^{2}(d w)$, is bounded on $L^{q}(d w)$, invertible on $L^{q}(d w)$, and its inverse is of the form $f \mapsto \mathcal{T}_{1 / c_{\phi}} f$. Let $f \in L^{p}(d w) \cap L^{2}(d w)$. Using the Plancherel identity (2.12), we get

$$
\begin{aligned}
\|f\|_{L^{p}(d w)} & =\sup _{g \in \mathcal{S}\left(\mathbb{R}^{N}\right),\|g\|_{L^{p^{\prime}(d w)}} \leq 1}\left|\int_{\mathbb{R}^{N}} f(\mathbf{x}) \overline{g(\mathbf{x})} d w(\mathbf{x})\right| \\
& =\sup _{g \in \mathcal{S}\left(\mathbb{R}^{N}\right),\|g\|_{L^{p^{\prime}(d w)} \leq 1}}\left|\int_{\mathbb{R}^{N}} \mathcal{F} f(\xi) \overline{\mathcal{F} g(\xi)} d w(\xi)\right| \\
& =\sup _{g \in \mathcal{S}\left(\mathbb{R}^{N}\right),\|g\|_{L^{p^{\prime}(d w)}} \leq 1}\left|\int_{\mathbb{R}^{N}} \mathcal{F} f(\xi) \overline{\mathcal{F}\left(\mathcal{T}_{1 / c_{\phi}} g\right)(\xi)} c_{\phi}(\xi) d w(\xi)\right| .
\end{aligned}
$$

Note that all the integrals are convergent, since all the functions $f, g$ and $\mathcal{T}_{1 / c_{\phi}} g$ belong to $L^{2}(d w)$. From (6.12) and (7.1) we conclude

$$
\begin{aligned}
\|f\|_{L^{p}(d w)} & =\sup _{g \in \mathcal{S}\left(\mathbb{R}^{N}\right),\|g\|_{L^{p^{\prime}(d w)}} \leq 1} \int_{\mathbb{R}^{N}} \int_{0}^{\infty} t^{2}\left\langle\nabla_{k}\left(\phi_{t} * f\right)(\mathbf{x}), \nabla_{k}\left(\phi_{t} *\left(\mathcal{T}_{1 / c_{\psi}} g\right)\right)(\mathbf{x})\right\rangle \frac{d t}{t} d w(\mathbf{x}) \\
& \leq \sup _{g \in \mathcal{S}\left(\mathbb{R}^{N}\right),\|g\|_{L^{p^{\prime}(d w)}} \leq 1}\left\|S_{\nabla_{k}, \phi}(f)\right\|_{L^{p}(d w)}\left\|S_{\nabla_{k}, \phi}\left(\mathcal{T}_{1 / c_{\phi}} g\right)\right\|_{L^{p^{\prime}}(d w)} \\
& \leq C_{p^{\prime}} \sup _{g \in \mathcal{S}\left(\mathbb{R}^{N}\right),\|g\|_{L^{p^{\prime}(d w)}} \leq 1}\left\|S_{\nabla_{k}, \phi}(f)\right\|_{L^{p}(d w)}\left\|\mathcal{T}_{1 / c_{\phi}} g\right\|_{L^{p^{\prime}}(d w)} \\
& \leq C\left\|S_{\nabla_{k}, \phi}(f)\right\|_{L^{p}(d w)},
\end{aligned}
$$

which completes the proof of (1.6) for $f \in L^{p}(d w) \cap L^{2}(d w)$. In order to relax the additional assumption $f \in L^{2}(d w)$, we apply the following easy approximation argument. We take $f_{n} \in L^{2}(d w) \cap L^{p}(d w)$ such that $\lim _{n \rightarrow \infty}\left\|f-f_{n}\right\|_{L^{p}(d w)}=0$. Then

$$
\begin{aligned}
\|f\|_{L^{p}(d w)} & =\lim _{n \rightarrow \infty}\left\|f_{n}\right\|_{L^{p}(d w)} \leq C \limsup _{n \rightarrow \infty}\left\|S_{\nabla_{k}, \phi}\left(f_{n}\right)\right\|_{L^{p}(d w)} \\
& \leq C^{\prime} \limsup _{n \rightarrow \infty}\left\|S_{\nabla_{k}, \phi}\left(f_{n}-f\right)\right\|_{L^{p}(d w)}+C^{\prime}\left\|S_{\nabla_{k}, \phi}(f)\right\|_{L^{p}(d w)} \leq C^{\prime}\left\|S_{\nabla_{k}, \phi}(f)\right\|_{L^{p}(d w)},
\end{aligned}
$$

where in the last inequality we have used Theorem 1.1.

The proof of (1.7) is identical to that of (1.6) and uses (6.13). Now (1.8) follows from (1.6), since $S_{\nabla_{k}, \phi} f(x) \leq C \mathfrak{g}_{\Gamma, \phi} f(\mathbf{x})$, see $(6.7)$.

Finally we prove Corollary 1.3. By direct calculations we have

$$
\left(t \frac{d}{d t} \phi_{t}\right)(\mathbf{x})=-\mathbf{N} \phi_{t}(\mathbf{x})-\sum_{j=1}^{N} t^{-\mathbf{N}} \frac{x_{j}}{t}\left(\partial_{j} \phi\right)(\mathbf{x} / t)=\psi_{t}(\mathbf{x}),
$$

where

$$
\psi(\mathbf{x})=-\mathbf{N} \phi(\mathbf{x})-\sum_{j=1}^{N} x_{j}\left(\partial_{j} \phi\right)(\mathbf{x}) .
$$

Clearly, $\psi \in C^{2 s}\left(\mathbb{R}^{N}\right)$ and satisfies (1.3). Moreover, by (2.3), we get

$$
\int_{\mathbb{R}^{N}}\left(t \frac{d}{d t} \phi_{t}\right)(\mathbf{x}) d w(\mathbf{x})=t \frac{d}{d t} \int_{\mathbb{R}^{N}} \phi_{t}(\mathbf{x}) d w(\mathbf{x})=0
$$

so $\int_{\mathbb{R}^{N}} \psi(\mathbf{x}) d w(\mathbf{x})=0$. Consequently,

$$
S_{\nabla_{t}, \phi} f(\mathbf{x})=S_{\psi} f(\mathbf{x})
$$


and (1.10) follows by Theorem 1.1. To prove (1.11), we note that for any $t_{1}>0$ and $\xi \in \mathbb{R}^{N}, \xi \neq 0$, we have

$$
\int_{t_{1}}^{\infty}(\mathcal{F} \psi)(t \xi) \frac{d t}{t}=\int_{t_{1}}^{\infty}\left(\mathcal{F} \psi_{t}\right)(\xi) \frac{d t}{t}=\int_{t_{1}}^{\infty} \frac{d}{d t}\left(\mathcal{F} \phi_{t}\right)(\xi) d t=-\mathcal{F} \phi\left(t_{1} \xi\right) .
$$

Since $(\mathcal{F} \phi)$ satisfies $(1.5)$, there is $t_{1}>0$ such that $\int_{t_{1}}^{\infty}(\mathcal{F} \psi)(t \xi) \frac{d t}{t} \neq 0$. So $(\mathcal{F} \psi)$ is not identically zero along the direction of $\xi$. Thus (1.11) follows from (7.2) and Theorem 1.2.

\section{REFERENCES}

[1] J.-Ph. Anker, J. Dziubański, A. Hejna, Harmonic functions, conjugate harmonic functions and the Hardy space $H^{1}$ in the rational Dunkl setting, J. Fourier Anal. Appl. 25 (2019), 2356-2418.

[2] B. Amri, A. Hammi, Dunkl-Schrödinger operators, Complex Anal. Oper. Theory (2018).

[3] M.F.E. de Jeu, The Dunkl transform, Invent. Math. 113 (1993), 147-162.

[4] M. de Jeu, M. Rösler, Asymptotic analysis for the Dunkl kernel, J. Approx. Theory 119 (2002), no. $1,110-126$.

[5] C.F. Dunkl, Reflection groups and orthogonal polynomials on the sphere, Math. Z. 197 (1988), no. $1,33-60$.

[6] C.F. Dunkl, Differential-difference operators associated to reflection groups, Trans. Amer. Math. 311 (1989), no. 1, 167-183.

[7] C.F. Dunkl, Hankel transforms associated to finite reflection groups, in: Proc. of the special session on hypergeometric functions on domains of positivity, Jack polynomials and applications, Proceedings, Tampa 1991, Contemp. Math. 138 (1989), 123-138.

[8] C.F. Dunkl, Integral kernels with reflection group invariance, Canad. J. Math. 43 (1991), no. 6, 1213-1227.

[9] J. Duoandikoetxea, Fourier Analysis, Graduate Studies in Mathematics, 29. American Mathematical Society, Providence, RI, 2001. xviii+222 pp. ISBN: 0-8218-2172-542-01.

[10] J. Dziubański and A. Hejna, Hörmander's multiplier theorem for the Dunkl transform, Journal of Functional Analysis 277 (2019), 2133-2159.

[11] P. Graczyk, T. Luks, M. Rösler, On the Green Function and Poisson Integrals of the Dunkl Laplacian, Potential Anal. 48 (2018), no. 3, 337360.

[12] L. Grafakos, Classical Fourier Analysis, 3rd edition, Graduate Texts in Mathematics, 249. Springer, New York, 2014.

[13] J. Liao, X. Zhang, Z. Li, On Littlewood-Paley functions associated with the Dunkl operator, Bull. Aust. Math. Soc. 96 (2017), no. 1, 126-138.

[14] H. Li and M. Zhao, Square function estimates for Dunkl operators, [arXiv:2003.11843].

[15] M. Rösler, Generalized Hermite polynomials and the heat equation for Dunkl operators, Comm. Math. Phys. 192 (1998), 519-542.

[16] M. Rösler, Positivity of Dunkl's intertwining operator, Duke Math. J. 98 (1999), no. 3, $445-463$.

[17] M. Rösler, A positive radial product formula for the Dunkl kernel, Trans. Amer.Math. Soc. 355 (2003), no. 6, 2413-2438.

[18] M. Rösler: Dunkl operators (theory and applications). In: Koelink, E., Van Assche, W. (eds.) Orthogonal polynomials and special functions (Leuven, 2002), 93-135. Lect. Notes Math. 1817, Springer-Verlag (2003).

[19] M. Rösler, M. Voit, Dunkl theory, convolution algebras, and related Markov processes, in Harmonic and stochastic analysis of Dunkl processes, P. Graczyk, M. Rösler, M. Yor (eds.), 1-112, Travaux en cours 71, Hermann, Paris, 2008.

[20] F. Soltani, Littlewood-Paley operators associated with the Dunkl operator on $\mathbb{R}$, J. Funct. Anal. 221 (2005), no. 1, 205-225.

[21] F. Soltani, Littlewood-Paley $g$-function in the Dunkl analysis on $\mathbb{R}^{d}$, JIPAM. J. Inequal. Pure Appl. Math. 6 (2005), no. 3, Article 84, 13 pp.

[22] E.M. Stein, Singular integral and differentiability properties of functions, Princeton Math. Series 30, Princeton Univ. Press, 1970.

[23] E.M. Stein, Harmonic analysis (real variable methods, orthogonality and oscillatory integrals), Princeton Math. Series 43, Princeton Univ. Press, 1993. 
[24] S. Thangavelu, Y. Xu, Convolution operator and maximal function for the Dunkl transform, J. Anal. Math. 97 (2005), 25-55.

[25] A. Velicu, Sobolev-Type Inequalities for Dunkl Operators, J. Funct. Anal. 279 (2020), no. 7, 108695, $37 \mathrm{pp}$.

[26] Ch. Yacoub, personal communication.

J. Dziubański and A. Hejna, Uniwersytet WrocŁawski, Instytut Matematyczny, Pl. Grunwaldzki 2/4, 50-384 WrocŁaW, Poland

E-mail address: jdziuban@math.uni.wroc.pl

E-mail address: hejna@math.uni.wroc.pl 